\title{
WHICH ERGODIC AVERAGES HAVE FINITE ASYMPTOTIC VARIANCE? ${ }^{1}$
}

\author{
By George Deligiannidis* AND ANTHONy LeE ${ }^{\dagger, \ddagger}, \S$ \\ University of Oxford ${ }^{*}$, University of Warwick ${ }^{\dagger}$, University of Bristol ${ }^{\ddagger}$ and \\ The Alan Turing Institute ${ }^{\S}$
}

\begin{abstract}
We show that the class of $L^{2}$ functions for which ergodic averages of a reversible Markov chain have finite asymptotic variance is determined by the class of $L^{2}$ functions for which ergodic averages of its associated jump chain have finite asymptotic variance. This allows us to characterize completely which ergodic averages have finite asymptotic variance when the Markov chain is an independence sampler. From a practical perspective, the most important result identifies a simple sufficient condition for all ergodic averages of $L^{2}$ functions of the primary variable in a pseudo-marginal Markov chain to have finite asymptotic variance.
\end{abstract}

1. Introduction. On a measurable space $(E, \mathcal{E})$, let $\Phi:=\left(\Phi_{n}\right)_{n \in \mathbb{N}}$ be an ergodic, reversible, discrete time Markov chain with Markov transition kernel $\Pi$ and invariant probability measure $\mu$. By ergodic, we mean $\Phi$ is $\mu$-irreducible; it need not be aperiodic. Such chains are often simulated on a computer for the purpose of computing Monte Carlo approximations of integrals $\mu(f):=\int_{\mathrm{E}} f(x) \mu(\mathrm{d} x)$, where $f \in L^{1}(\mathrm{E}, \mu):=\{g: \mu(|g|)<\infty\}$. Ergodic averages, $n^{-1} \sum_{i=1}^{n} f\left(\Phi_{i}\right)$, associated with such Markov chains converge almost surely as $n \rightarrow \infty$ to $\mu(f)$ for $\mu$-almost all $\Phi_{1}$ and all $f \in L^{1}(\mathrm{E}, \mu)$ [see, e.g., Meyn and Tweedie (2009), Chapter 17], and are frequently used to approximate intractable integrals in computer science, physics and statistics. The behaviour of such approximations is now quite well understood, and central limit theorems (CLTs) for rescaled ergodic averages and quantitative bounds on their asymptotic variance have been established in a number of settings. We define the asymptotic variance of ergodic averages of a function $f \in L^{2}(\mathrm{E}, \mu):=\left\{g: \mu\left(g^{2}\right)<\infty\right\}$ to be

$$
\operatorname{var}(f, \Pi):=\lim _{n \rightarrow \infty} n \operatorname{var}\left\{\frac{1}{n} \sum_{i=1}^{n} f\left(\Phi_{i}\right)\right\}, \quad \Phi_{1} \sim \mu .
$$

For ergodic, $\mu$-reversible Markov chains, this limit exists for all $f \in L^{2}(\mathrm{E}, \mu)$ but may be infinite. Denoting the function $x \mapsto f(x)-c$ by $f-c$, where $c$ is

Received July 2016; revised May 2017.

${ }^{1}$ Supported by The Alan Turing Institute under the EPSRC grant EP/N510129/1.

MSC2010 subject classifications. 60J05, 60J22, 60F05, 65C40.

Key words and phrases. Markov chain Monte Carlo, asymptotic variance, jump chain, independent Metropolis-Hastings, pseudo-marginal method. 
a constant, we observe that $\operatorname{var}(f, \Pi)=\operatorname{var}(f-c, \Pi)$, and so one can restrict consideration to zero-mean functions $f \in L_{0}^{2}(\mathrm{E}, \mu):=\left\{g \in L^{2}(\mathrm{E}, \mu): \mu(g)=0\right\}$ without loss of generality.

A strong qualitative property of a Markov chain is that it is variance bounding [Roberts and Rosenthal (2008)]: if $\Phi$ is variance bounding, then it satisfies

$$
\sup _{f \in L_{0}^{2}(\mathrm{E}, \mu), \operatorname{var}_{\mu}(f)=1} \operatorname{var}(f, \Pi)<\infty,
$$

where $\operatorname{var}_{\mu}(f)$ is the variance of $f\left(\Phi_{1}\right)$ when $\Phi_{1} \sim \mu$. For reversible Markov chains, variance bounding is closely related to geometric ergodicity and equivalent to finite $\operatorname{var}(f, \Pi)$ for all $f \in L^{2}(\mathrm{E}, \mu)$. By Kipnis and Varadhan (1986), this implies a $\sqrt{n}$-CLT for all $f \in L^{2}(\mathrm{E}, \mu)$ with limiting variance equal to the asymptotic variance, that is, $n^{-1 / 2} \sum_{i=1}^{n}\left[f\left(\Phi_{i}\right)-\mu(f)\right]$ converges weakly to a $N(0, \operatorname{var}(f, \Pi))$ random variable when $\Phi_{1} \sim \mu$. Hence, variance bounding provides some qualitative assurance of the practicality of using ergodic averages as approximations of $\mu(f)$ for all $f$ such that $\operatorname{var}_{\mu}(f)<\infty$.

Some Markov chains used in practice are ergodic and reversible but not variance bounding, so for at least some $f \in L_{0}^{2}(\mathrm{E}, \mu), \operatorname{var}(f, \Pi)$ is not finite: the proof of Theorem 7 of Roberts and Rosenthal (2008) constructs one such $f$. On such occasions, it is beneficial to have some guarantees on the subset of $L_{0}^{2}(\mathrm{E}, \mu)$ whose ergodic averages do have finite asymptotic variance. Relevant results in this spirit include Theorems 4.1-4.5 of Jarner and Roberts (2002), Theorem 2 of Jarner and Roberts (2007) and Theorem 4.1 of Bednorz, Łatuszyński and Latała (2008), involving the verification of Foster-Lyapunov drift criteria and/or regenerative properties of $\Phi$. We note, however, that these results concern explicitly the existence of a $\sqrt{n}$-CLT with finite limiting variance rather than finiteness of the asymptotic variance.

In this paper, we consider the class of $\pi$-reversible, ergodic Markov chains $X:=$ $\left(X_{n}\right)_{n \in \mathbb{N}}$ evolving on $\mathrm{E}$ whose Markov transition kernel is of the form

$$
P(x, A):=\varrho(x) \tilde{P}(x, A)+[1-\varrho(x)] \mathbf{1}_{A}(x), \quad A \in \mathcal{E},
$$

where $\tilde{P}$ is the Markov transition kernel of a reversible Markov chain $\tilde{X}:=$ $\left(\tilde{X}_{n}\right)_{n \in \mathbb{N}}$, and $\varrho: E \rightarrow(0,1]$. Such chains arise frequently in statistical applications, Metropolis-Hastings chains being a notable example. We will refer to $\tilde{X}$ as the jump chain associated with $X$ and $\tilde{P}$ the jump kernel associated with $P$. The invariant probability measure $\tilde{\pi}$ associated with $\tilde{X}$ is related to $\pi$ through $\varrho$ and defined in Section 2. Jump chains are a recurring theme in the study of Markov processes. For example, continuous time jump Markov processes are often defined in terms of a jump chain and a Poisson process describing the holding times [see, e.g., Pardoux (2008), Chapter 7], which allows the use of discretetime techniques in their analysis. In the study of piecewise deterministic Markov processes, properties like recurrence, geometric ergodicity can often be deduced 
from those of the jump chain, or an embedded chain more generally, which are often simpler to derive [see, e.g., Costa (1990), Costa and Dufour (2008)]. In the context of Markov chain Monte Carlo, jump chains have been studied by Douc and Robert (2011) and Doucet et al. (2015), but for different purposes than here. Denoting $x \mapsto f(x) / \varrho(x)$ by $f / \varrho$, our first main result is that for $f \in L_{0}^{2}(\mathrm{E}, \pi)$, $\operatorname{var}(f, P)<\infty$ if and only if $f / \varrho \in L_{0}^{2}(\mathrm{E}, \tilde{\pi})$ and $\operatorname{var}(f / \varrho, \tilde{P})<\infty$, extending a result by Doucet et al. (2015). This equivalence is interesting because it allows us to infer that when $\tilde{P}$ is variance bounding, then those functions $f$ such that $f / \varrho \in L_{0}^{2}(\mathrm{E}, \tilde{\pi})$ are exactly the functions in $L_{0}^{2}(\mathrm{E}, \pi)$ for which $\operatorname{var}(f, P)<\infty$.

We apply this result to independent Metropolis-Hastings (IMH) Markov chains as well as pseudo-marginal Markov chains. When $P$ is an IMH kernel, we characterize the class of $\pi$-integrable functions satisfying $\operatorname{var}(f, P)<\infty$. To the best of our knowledge, this is the first result of this kind for independence samplers. Pseudo-marginal Markov chains [Lin, Liu and Sloan (2000), Beaumont (2003), Andrieu and Roberts (2009)] are a Monte Carlo innovation that has received considerable recent attention. When $P$ is a pseudo-marginal kernel, $X$ is a $\pi$-reversible Markov chain evolving on $E=X \times \mathbb{R}_{+}$, where $\pi$ admits as a marginal the invariant distribution of a $\bar{\pi}$-reversible, "marginal" Markov chain $\bar{X}$ evolving on $\mathrm{X}$. The extension of the state space accommodates the inclusion of what can be viewed as a multiplicative noise variable, and simulating $X$ is in many respects like simulating a noisy version of $\bar{X}$. The noise introduced is of great practical importance: computationally one can simulate $X$ in some cases where one cannot simulate $\bar{X}$, while the properties of the noise variables introduced affect in a variety of ways the behaviour of $X$ and associated ergodic averages. A brief summary of relevant results in this active area of research can be found in Section 4. Our main contribution in this context is Theorem 3 , which gives a simple, sufficient condition for all ergodic averages of functions $f(\cdot, u)=f_{X} \in L_{0}^{2}(\mathrm{X}, \bar{\pi})$ to have $\operatorname{var}(f, P)<\infty$ when $\bar{X}$ is variance bounding. This condition is both necessary and sufficient in some settings, but not in general, and amounts to uniformly bounded second moments of the noise variables. This complements existing results, and in particular we do not make explicit assumptions about $\bar{X}$ beyond assuming it is variance bounding. In contrast, previous sufficient conditions when $X$ is not itself variance bounding have been found when the marginal chain is strongly uniformly ergodic, or under fairly specific assumptions on $\bar{X}$ [Andrieu and Vihola (2016), Remark 15].

We close this section with some notation and definitions. $\mathbb{N}$ denotes the positive integers, $\mathbb{R}_{+}$the nonnegative reals. For $v$ a measure on a measurable space $(\mathrm{S}, \mathcal{S})$, and $f$ a measurable function, we denote $v(f):=\int_{\mathrm{S}} f(x) v(\mathrm{~d} x)$. We define $L^{2}(\mathrm{~S}, v)=\left\{f: v\left(f^{2}\right)<\infty\right\}$ and $L_{0}^{2}(\mathrm{~S}, v):=\left\{f \in L^{2}(\mathrm{~S}, v): v(f)=0\right\}$. Similarly, $L^{1}(\mathrm{~S}, v)=\{f: v(|f|)<\infty\}$ and $L_{0}^{1}(\mathrm{~S}, v):=\left\{f \in L^{1}(\mathrm{~S}, v): v(f)=0\right\}$. For functions $f, g: \mathrm{S} \rightarrow \mathbb{R}$, we write $f \cdot g$ for the function $x \mapsto f(x) g(x)$ and when $g$ is strictly positive $f / g$ for the function $x \mapsto f(x) / g(x)$. For a $\mu$-reversible Markov kernel $\Pi$, we will say $\Pi$ is variance bounding when its associated Markov 
chain is variance bounding. We write $\wedge$ and $\vee$ to denote min and max, respectively. When we refer to a geometric distribution, we mean the distribution on $\mathbb{N}$.

Many of our results rely on Dirichlet forms and the variational definition of the right spectral gap of a Markov operator. For a generic measurable space $(\mathrm{S}, \mathcal{S})$ and measure $\mu$, we denote by $\langle\cdot, \cdot\rangle_{\mu}$ the inner product on $L^{2}(\mathrm{~S}, \mu)$. We often rely on viewing a $\mu$-reversible Markov kernel $\Pi$ as a self-adjoint operator on $L^{2}(\mathrm{~S}, \mu)$ or $L_{0}^{2}(\mathrm{~S}, \mu)$; this should always be clear from the context. We define the Dirichlet form of a such a Markov kernel, for $f \in L^{2}(\mathrm{~S}, \mu)$ as

$$
\mathcal{E}_{\Pi}(f):=\langle f,(I-\Pi) f\rangle_{\mu}=\frac{1}{2} \int_{\mathrm{S}} \mu(\mathrm{d} x) \Pi(x, \mathrm{~d} y)[f(y)-f(x)]^{2} .
$$

The right spectral gap of $\Pi$, as an operator on $L_{0}^{2}(\mathrm{~S}, \mu)$, is usually defined as the distance of the right end of the spectrum from 1, or equivalently [Yosida (1980), Theorem 2, page 320] as

$$
\operatorname{Gap}(\Pi):=\inf _{f \in L_{0}^{2}(\mathrm{~S}, \mu),\langle f, f\rangle_{\mu}=1} \mathcal{E}_{\Pi}(f)=\inf _{f \in L^{2}(\mathrm{~S}, \mu), \operatorname{var}_{\mu}(f)>0} \frac{\mathcal{E}_{\Pi}(f)}{\operatorname{var}_{\mu}(f)},
$$

where the rightmost infimum can be taken over $L_{0}^{2}(\mathrm{~S}, \mu)$ or $L^{2}(\mathrm{~S}, \mu)$. From Theorem 14 of Roberts and Rosenthal (2008), $\Pi$ is variance bounding if and only if $\operatorname{Gap}(\Pi)>0$.

2. Relationship between $\boldsymbol{X}$ and $\tilde{X}$. We describe briefly the relationship between the chain $X$ and its associated jump chain $\tilde{X}$, following Douc and Robert (2011). Since $X$ is $\pi$-reversible, it is straightforward to establish that $\tilde{X}$ is an ergodic, $\tilde{\pi}$-reversible Markov chain, where

$$
\tilde{\pi}(\mathrm{d} x)=\pi(\mathrm{d} x) \varrho(x) / \pi(\varrho),
$$

from which it follows that $\pi(f)=\pi(\varrho) \tilde{\pi}(f / \varrho)$. One can construct a realization of $X$ from $\tilde{X}$ by introducing a sequence of holding times $\left(\tau_{n}\right)_{n \in \mathbb{N}}$, where for each $n \in \mathbb{N}, \tau_{n}$ is conditionally independent of all other random variables given $\tilde{X}_{n}$ with $\tau_{n} \mid\left\{\tilde{X}_{n}=x\right\} \sim \operatorname{Geometric}(\varrho(x))$. By defining $S_{n}:=\inf \left\{k \geq 1: \sum_{i=1}^{k} \tau_{i} \geq n\right\}$ for $n \in \mathbb{N}$, one can verify that $\left(\tilde{X}_{S_{n}}\right)_{n \in \mathbb{N}}$ is a realization of $X$ with initial state $\tilde{X}_{1}$.

Our first main result is the following, the converse part of which is the novel addition to Proposition 2 of Doucet et al. (2015). The relation (4) may seem obvious. Indeed, if one assumes that $\operatorname{var}(f, P)$ and $\operatorname{var}(f / \rho, \tilde{P})$ are both finite, then (4) follows from the representation of $X$ in terms of $\tilde{X}$ and a careful application of the Kipnis-Varadhan CLT [Kipnis and Varadhan (1986)], as in the proof of Proposition 2 in Doucet et al. (2015). The main difficulty lies in proving the first part of the theorem, where the path-wise relation between $X$ and $\tilde{X}$ does not offer much traction without further restrictive assumptions. 
TheOREM 1. Let $f \in L_{0}^{2}(\mathrm{E}, \pi)$. Then $\operatorname{var}(f, P)<\infty \Longleftrightarrow f / \varrho \in L_{0}^{2}(\mathrm{E}, \tilde{\pi})$ and $\operatorname{var}(f / \varrho, \tilde{P})<\infty$. Moreover,

$$
\operatorname{var}(f, P)=\pi\left(f^{2} / \varrho\right)-\pi\left(f^{2}\right)+\pi(\varrho) \operatorname{var}(f / \varrho, \tilde{P}) .
$$

PROOF. The direction $(\Rightarrow)$ and the expression for the variance is Proposition 2 of Doucet et al. (2015). We provide here the proof of $(\Leftarrow)$. We recall the variational expression for the asymptotic variance associated with a $\mu$-reversible Markov kernel $\Pi$ suggested by Caracciolo, Pelissetto and Sokal (1990), and discussed in Section 4 of Andrieu and Vihola (2016),

(5) $\operatorname{var}(f, \Pi)=2\left[\sup _{g \in L^{2}(\mathrm{E}, \mu)} 2\langle f, g\rangle_{\mu}-\mathcal{E}_{\Pi}(g)\right]-\langle f, f\rangle_{\mu}, \quad f \in L_{0}^{2}(\mathrm{E}, \mu)$.

We observe from (1) that for $g \in L^{2}(\mathrm{E}, \pi)$,

$$
\mathcal{E}_{P}(g)=\frac{1}{2} \int_{\mathrm{E}} \pi(\mathrm{d} x) \varrho(x) \tilde{P}(x, \mathrm{~d} y)[g(y)-g(x)]^{2}=\pi(\varrho) \mathcal{E}_{\tilde{P}}(g)
$$

and that $\langle f / \varrho, g\rangle_{\tilde{\pi}}=\langle f, g\rangle_{\pi} / \pi(\varrho)$. Let $f / \varrho \in L_{0}^{2}(\mathrm{E}, \tilde{\pi})$, which implies $f \in$ $L_{0}^{2}(\mathrm{E}, \pi)$. Since $L^{2}(\mathrm{E}, \pi) \subseteq L^{2}(\mathrm{E}, \tilde{\pi})$, and using $(6)$,

$$
\begin{aligned}
\sup _{g \in L^{2}(\mathrm{E}, \tilde{\pi})} 2\langle f / \varrho, g\rangle_{\tilde{\pi}}-\mathcal{E}_{\tilde{P}}(g) & \geq \sup _{g \in L^{2}(\mathrm{E}, \pi)} 2\langle f / \varrho, g\rangle_{\tilde{\pi}}-\mathcal{E}_{\tilde{P}}(g) \\
& =\frac{1}{\pi(\varrho)}\left[\sup _{g \in L^{2}(\mathrm{E}, \pi)} 2\langle f, g\rangle_{\pi}-\mathcal{E}_{P}(g)\right] .
\end{aligned}
$$

Combining this bound with the expressions for $\operatorname{both} \operatorname{var}(f / \varrho, \tilde{P})$ and $\operatorname{var}(f, P)$ using (5), we obtain

$$
\operatorname{var}(f, P) \leq \pi(\varrho) \operatorname{var}(f / \varrho, \tilde{P})+\pi\left(f^{2} / \varrho\right)-\pi\left(f^{2}\right),
$$

so $f / \varrho \in L_{0}^{2}(\mathrm{E}, \tilde{\pi})$ and $\operatorname{var}(f / \varrho, \tilde{P})<\infty \Rightarrow \operatorname{var}(f, P)<\infty$.

REMARK 1. A different proof of Theorem 1 can also be obtained through the analysis of the multiplication operator $T: f \mapsto f / \rho$ between the Hilbert spaces $\left(L_{0}^{2}(\mathrm{E}, \pi),\langle\cdot, \cdot\rangle_{1}\right)$ and $\left(L_{0}^{2}(\mathrm{E}, \tilde{\pi}),\langle\cdot, \cdot\rangle_{2}\right)$, where

$$
\begin{aligned}
& \langle f, g\rangle_{1}:=\left\langle(I-P)^{-1 / 2} f,(I-P)^{-1 / 2} g\right\rangle_{\pi}, \\
& \langle f, g\rangle_{2}:=\pi(\rho)\left\langle(I-\tilde{P})^{-1 / 2} f,(I-\tilde{P})^{-1 / 2} g\right\rangle_{\tilde{\pi}} .
\end{aligned}
$$

In the process of showing that $T$ is invertible and, therefore, proving Theorem 1, one also obtains the interesting fact that $T$ as defined is in fact an isometry, that is,

$$
\langle f, f\rangle_{1}=\langle f / \rho, f / \rho\rangle_{2} .
$$

This proves (4) directly, without requiring a careful application of the CLT as was done in the proof of Proposition 2 in Doucet et al. (2015). 
COROllary 1. If $\tilde{P}$ is variance bounding and $f \in L_{0}^{2}(\mathrm{E}, \pi)$, then $\operatorname{var}(f, P)<\infty \Longleftrightarrow f / \varrho \in L_{0}^{2}(\mathrm{E}, \tilde{\pi})$.

The following example illustrates one way this result can be applied.

EXAMPLE. Let $p<1 / 2$ and $\varrho: \mathbb{N} \rightarrow(0,1]$, and consider the reversible Markov chain $X$ on $\mathbb{N}$ with $P(1,1)=1-\varrho(1) p, P(1,2)=\varrho(1) p$ and for $x>1$, $P(x, x)=1-\varrho(x), P(x, x+1)=\varrho(x) p$ and $P(x, x-1)=\varrho(x)(1-p)$. The jump chain $\tilde{X}$ is a simple random walk on $\mathbb{N}$ with $\tilde{\pi}$ the Geometric $(1-p /[1-p])$ distribution, and since $p<1 / 2$ it is variance bounding [see, e.g., Meyn and Tweedie (2009), Section 15.5.1]. We have $\pi(x) \propto[p /(1-p)]^{x} / \varrho(x)$ and it can be shown that $X$ is variance bounding if and only if $\inf _{x \in X} \varrho(x)>0$. Irrespective of this, Corollary 1 implies that the functions $f \in L_{0}^{2}(\mathbb{N}, \pi)$ that have $\operatorname{var}(f, P)<\infty$ are those satisfying $\sum_{x \in \mathbb{N}}[p /(1-p)]^{x} f(x)^{2} / \varrho(x)^{2}<\infty$.

The following proposition states that $\tilde{P}$ inherits variance bounding from $P$. The example above shows that the converse clearly does not hold, and this is why Corollary 1 provides a route to the characterization of functions whose ergodic averages have finite asymptotic variance.

\section{Proposition 1. $P$ and $\tilde{P}$ satisfy $\operatorname{Gap}(\tilde{P}) \geq \operatorname{Gap}(P)$.}

Proof. If $\operatorname{Gap}(P)=0$, then the result is trivial. If $\operatorname{Gap}(P)>0$, then $\varrho^{*}:=$ $\pi$-essinf $\varrho>0$ by Theorem 1 of Lee and Latuszyński (2014). It follows that $L^{2}(\mathrm{E}, \tilde{\pi})=L^{2}(\mathrm{E}, \pi)$. If $g \in L_{0}^{2}(\mathrm{E}, \pi)$,

$$
\frac{\operatorname{var}_{\pi}(g)}{\operatorname{var}_{\tilde{\pi}}(g)}=\frac{\pi\left(g^{2}\right)}{\tilde{\pi}\left(g^{2}\right)-\tilde{\pi}(g)^{2}} \geq \frac{\pi\left(g^{2}\right)}{\tilde{\pi}\left(g^{2}\right)}=\frac{\pi\left(g^{2}\right) \pi(\varrho)}{\pi\left(\varrho \cdot g^{2}\right)} \geq \pi(\varrho),
$$

and so $\operatorname{var}_{\pi}(g) / \operatorname{var}_{\tilde{\pi}}(g) \geq \pi(\varphi)$ for any $g \in L^{2}(\mathrm{E}, \pi)$. From (6), for any $g \in$ $L^{2}(\mathrm{E}, \tilde{\pi})=L^{2}(\mathrm{E}, \pi)$,

$$
\frac{\mathcal{E}_{\tilde{P}}(g)}{\operatorname{var}_{\tilde{\pi}}(g)}=\frac{\mathcal{E}_{P}(g) / \pi(\varrho)}{\operatorname{var}_{\pi}(g)} \cdot \frac{\operatorname{var}_{\pi}(g)}{\operatorname{var}_{\tilde{\pi}}(g)} \geq \frac{\mathcal{E}_{P}(g)}{\operatorname{var}_{\pi}(g)},
$$

and it follows from (2) and taking infima that $\operatorname{Gap}(\tilde{P}) \geq \operatorname{Gap}(P)$.

Remark 2. One can deduce from Theorem 1 that for $f \in L_{0}^{2}(\mathrm{E}, \pi)$,

$$
\operatorname{var}(\tilde{f}, \tilde{P})=\pi(\varrho)^{2} \operatorname{var}(f / \varrho, \tilde{P})=\pi(\varrho)\left[\operatorname{var}(f, P)-\pi\left(f^{2} / \varrho\right)+\pi\left(f^{2}\right)\right],
$$

where $\tilde{f}:=\pi(\varrho) \cdot f / \varrho$. It follows that the sequence of approximations of $\pi(f)$ defined by $n^{-1} \sum_{i=1}^{n} \tilde{f}\left(\tilde{X}_{i}\right)$ has an asymptotic variance at least $\pi(\varrho)$ smaller than $n^{-1} \sum_{i=1}^{n} f\left(X_{i}\right)$. In the context of limiting variances for associated central limit theorems, rather than the asymptotic variances studied here, this observation is one of the main contributions and motivations for Douc and Robert (2011), which we will return to in Section 5. 
In the sequel, we will apply Theorem 1 exclusively to the case where

$$
P(x, A)=\int_{A} q(x, \mathrm{~d} y) \alpha(x, y)+[1-\varrho(x)] \mathbf{1}_{A}(x), \quad A \in \mathcal{E},
$$

with $q$ a Markov kernel, $\alpha: \mathrm{E}^{2} \rightarrow[0,1]$ an acceptance probability function and $\varrho(x):=\int_{E} q(x, \mathrm{~d} y) \alpha(x, y)$ denoting the probability of accepting a proposal from $q(x, \cdot)$. In this case, the jump kernel $\tilde{P}$ is

$$
\tilde{P}(x, A)=\int_{A} q(x, \mathrm{~d} y) \alpha(x, y) / \varrho(x), \quad A \in \mathcal{E},
$$

and $\tilde{X}$ is the Markov chain of accepted proposals. A particular $\alpha$, which guarantees $\pi$-reversibility of $P$, is the Metropolis-Hastings acceptance probability function [Metropolis et al. (1953), Hastings (1970)]

$$
\alpha(x, y)=1 \wedge \frac{\pi(\mathrm{d} y) q(y, \mathrm{~d} x)}{\pi(\mathrm{d} x) q(x, \mathrm{~d} y)} .
$$

\section{Independent Metropolis-Hastings.}

3.1. Characterization of functions with finite asymptotic variance. We now apply Theorem 1 to characterize those $f \in L_{0}^{2}(\mathrm{E}, \pi)$ with finite $\operatorname{var}(f, P)$ when $P$ is an IMH kernel. In fact, we are able to characterize those $f \in L_{0}^{1}(\mathrm{E}, \pi)$ with finite $\operatorname{var}(f, P)$ in this specific case. An IMH kernel is a Metropolis-Hastings kernel where in (7), $q(x, \cdot)=\mu(\cdot)$ for all $x \in \mathrm{E}$, where $\mu$ is a probability measure such that $\pi \ll \mu$. The acceptance probability (8) is

$$
\alpha(x, y):=1 \wedge \frac{w(y)}{w(x)}, \quad x, y \in \mathrm{E}, \text { where } w:=\frac{\mathrm{d} \pi}{\mathrm{d} \mu} .
$$

The resulting IMH chain $X$ has been analyzed for various $\pi$ and $\mu$. For example, Tierney (1994) noted that when $\bar{w}:=\pi$-ess sup $w<\infty, X$ is uniformly ergodic with a spectral gap of $1 / \bar{w}$, and Mengersen and Tweedie (1996) showed that when $\bar{w}=\infty, X$ is not even geometrically ergodic. In Jarner and Roberts (2002) and Jarner and Roberts (2007), conditions guaranteeing polynomial ergodicity of $X$, and hence finite associated asymptotic variances for some functions, are obtained under assumptions on $\pi$ and $\mu$. Using Theorem 1, however, we are able to characterize exactly the class of functions with finite associated asymptotic variances.

THEOREM 2. Let $f \in L_{0}^{1}(\mathrm{E}, \pi)$. For the $I M H$, $\operatorname{var}(f, P)<\infty$ if and only if $f \in L_{0}^{2}(\mathrm{E}, \pi)$ and $w \cdot f \in L_{0}^{2}(\mathrm{E}, \mu)$.

Lemma 1 is used multiple times in our proofs. 
LEMMA 1. Let $Y$ be a nonnegative random variable with $\mathbb{E}[Y]=1$. Then

$$
\frac{1}{\mathbb{E}\left[Y^{2}\right]+c} \leq \mathbb{E}\left[1 \wedge \frac{Y}{c}\right] \leq 1 \wedge \frac{1}{c} .
$$

ProOF. For the upper bound, we have $\mathbb{E}\left[1 \wedge \frac{Y}{c}\right] \leq 1 \wedge \mathbb{E}\left[\frac{Y}{c}\right]=1 \wedge \frac{1}{c}$. For the lower bound, letting $v$ be the probability measure associated with $Y$,

$$
\begin{aligned}
\mathbb{E}\left[1 \wedge \frac{Y}{c}\right] & =\int_{\mathbb{R}_{+}} v(\mathrm{~d} y)\left(1 \wedge \frac{y}{c}\right)=\int_{\mathbb{R}_{+}} v(\mathrm{~d} y) y\left(\frac{1}{y} \wedge \frac{1}{c}\right) \\
& \geq\left[\int_{\mathbb{R}_{+}} v(\mathrm{~d} y) y(y \vee c)\right]^{-1} \geq\left(\mathbb{E}\left[Y^{2}\right]+c\right)^{-1},
\end{aligned}
$$

where we have used the fact that $v(\mathrm{~d} y) y$ is also a probability measure, Jensen's inequality and $a \vee b \leq a+b$.

COROLlaRY 2. For the IMH,

$$
\frac{1}{\pi(w)+w(x)} \leq \varrho(x) \leq 1 \wedge \frac{1}{w(x)} .
$$

LEMMA 2. For the IMH, if $f \in L_{0}^{1}(\mathrm{E}, \pi) \backslash L_{0}^{2}(\mathrm{E}, \pi)$, then $\operatorname{var}(f, P)=\infty$.

Proof. Let $A:=\{x \in \mathrm{E}: f(x) \geq 0\}$ and $B:=\{x \in \mathrm{E}: f(x) \leq 0\}$. Since $f \in$ $L_{0}^{1}(\mathrm{E}, \pi) \backslash L_{0}^{2}(\mathrm{E}, \pi)$, at least one of $\pi\left(\mathbf{1}_{A} \cdot f^{2}\right)$ or $\pi\left(\mathbf{1}_{B} \cdot f^{2}\right)$ is infinite, so let $C \in\{A, B\}$ satisfy $\pi\left(\mathbf{1}_{C} \cdot f^{2}\right)=\infty$ and observe that $\mu(C)>0$ since $\pi \ll \mu$. We consider the event $\left(X_{1}, \ldots, X_{n}\right) \in C^{n}$, noting that for $x \in C, P(x, C) \geq \mu(C)$. On the event $\left(X_{1}, \ldots, X_{n}\right) \in C^{n}$, we have $\left[\sum_{i=1}^{n} f\left(X_{i}\right)\right]^{2} \geq f\left(X_{1}\right)^{2}$, and so for any $n \in \mathbb{N}$,

$$
\begin{aligned}
\operatorname{var}\left(\frac{1}{n} \sum_{i=1}^{n} f\left(X_{i}\right)\right) & =\mathbb{E}\left[\left\{\frac{1}{n} \sum_{i=1}^{n} f\left(X_{i}\right)\right\}^{2}\right] \\
& \geq \mathbb{E}\left[\mathbf{1}_{C^{n}}\left(X_{1}, \ldots, X_{n}\right) n^{-2} f\left(X_{1}\right)^{2}\right] \\
& \geq n^{-2} \mu(C)^{n-1} \pi\left(\mathbf{1}_{C} \cdot f^{2}\right)=\infty
\end{aligned}
$$

Hence $\operatorname{var}(f, P)$ is infinite.

LEMMA 3. For the IMH, $\tilde{P}$ satisfies the one-step minorization condition

$$
\tilde{P}(x, A) \geq \pi(\varrho) \tilde{\pi}(A), \quad x \in \mathrm{E}, A \in \mathcal{E},
$$

so $\tilde{X}$ is uniformly ergodic. Therefore, for $f \in L_{0}^{2}(\mathrm{E}, \pi), \operatorname{var}(f, P)<\infty \Longleftrightarrow$ $f / \varrho \in L_{0}^{2}(\mathrm{E}, \tilde{\pi})$. 
PROOF. Straightforward calculations and (3) provide

$$
\begin{aligned}
\tilde{P}(x, \mathrm{~d} y) & =\frac{\mu(\mathrm{d} y) \alpha(x, y)}{\varrho(x)}=\frac{\mu(\mathrm{d} y)\left[1 \wedge \frac{w(y)}{w(x)}\right]}{\varrho(x)} \\
& =\frac{\pi(\mathrm{d} y)\left[\frac{1}{w(y)} \wedge \frac{1}{w(x)}\right]}{\varrho(x)}=\frac{\tilde{\pi}(\mathrm{d} y) \pi(\varrho)\left[\frac{1}{w(y)} \wedge \frac{1}{w(x)}\right]}{\varrho(x) \varrho(y)} \\
& =\frac{\tilde{\pi}(\mathrm{d} y) \pi(\varrho)}{[w(y) \vee w(x)] \varrho(x) \varrho(y)} \geq \tilde{\pi}(\mathrm{d} y) \pi(\varrho),
\end{aligned}
$$

where in the inequality we have used symmetry and that when $w(x) \geq w(y)$, the upper bound in Corollary 2 implies that

$$
[w(y) \vee w(x)] \varrho(x) \varrho(y)=w(x) \varrho(x) \varrho(y) \leq \varrho(y) \leq 1 .
$$

For the second part, since $\tilde{X}$ is uniformly ergodic it is variance bounding, and the result follows from Corollary 1.

LEMMA 4. For the IMH, let $f \in L_{0}^{2}(\mathrm{E}, \pi)$. Then $f / \varrho \in L_{0}^{2}(\mathrm{E}, \tilde{\pi})$, if and only if $w \cdot f \in L_{0}^{2}(\mathrm{E}, \mu)$.

Proof. We note that $f / \varrho \in L_{0}^{2}(\mathrm{E}, \tilde{\pi}) \Longleftrightarrow \pi\left(f^{2} / \varrho\right)<\infty$. If $\pi\left(f^{2} / \varrho\right)<\infty$, then since $w(x) \leq 1 / \varrho(x)$ by Lemma 2 ,

$$
\mu\left(w^{2} \cdot f^{2}\right)=\pi\left(w \cdot f^{2}\right) \leq \pi\left(f^{2} / \varrho\right)<\infty .
$$

For the converse, assume $\pi\left(f^{2}\right)<\infty$ and $\mu\left(w^{2} \cdot f^{2}\right)=\pi\left(w \cdot f^{2}\right)<\infty$. We consider two cases: $\pi(w)<\infty$ and $\pi(w)=\infty$. If $\pi(w)<\infty$, then $\varrho(x) \geq$ $1 /[\pi(w)+w(x)]$ by Lemma 2 , so

$$
\pi\left(f^{2} / \varrho\right) \leq \pi(w) \pi\left(f^{2}\right)+\pi\left(w \cdot f^{2}\right)<\infty
$$

If $\pi(w)=\infty$, then for each $x \in \mathrm{X}$ we define the region of certain acceptance $A_{x}:=\{y: w(y) \geq w(x)\}$ and observe that

$$
\varrho(x)=\int_{\mathrm{E}} 1 \wedge \frac{w(y)}{w(x)} \mu(\mathrm{d} y)=\mu\left(A_{x}\right)+\pi\left(A_{x}^{\complement}\right) / w(x) .
$$

Since $\mu(w)=1, w$ is $\mu$-almost everywhere finite, and thus there exists a $C>0$ such that $B:=\{x: w(x) \geq C\}$ satisfies $\pi\left(B^{\complement}\right)>0$. For example, $C$ could be an appropriately chosen quantile of $w(X)$ when $X \sim \pi$. Moreover, since $\pi(w)=\infty$, we must have $\pi(B)>0$, which implies $\mu(B)>0$ since $\pi \ll \mu$. We observe that if $x \in B$,

$$
A_{x} \subseteq B \quad \Rightarrow \quad \pi\left(A_{x}^{\complement}\right) \geq \pi\left(B^{\complement}\right) \quad \Rightarrow \quad \varrho(x) \geq \pi\left(B^{\complement}\right) / w(x),
$$

while if $x \notin B$,

$$
A_{x} \supseteq B \quad \Rightarrow \quad \mu\left(A_{x}\right) \geq \mu(B) \quad \Rightarrow \quad \varrho(x) \geq \mu(B) .
$$


Therefore,

$\pi\left(f^{2} / \varrho\right)=\pi\left(\mathbf{1}_{B} \cdot f^{2} / \varrho\right)+\pi\left(\mathbf{1}_{B^{\complement}} \cdot f^{2} / \varrho\right) \leq \frac{\pi\left(\mathbf{1}_{B} \cdot w \cdot f^{2}\right)}{\pi\left(B^{\complement}\right)}+\frac{\pi\left(\mathbf{1}_{B^{\complement}} \cdot f^{2}\right)}{\mu(B)}<\infty$.

Proof of TheOrem 2. This is a consequence of Lemmas 2, 3 and 4.

REMARK 3. The characterization of $L_{0}^{1}(E, \pi)$ functions for which independence sampler ergodic averages have finite asymptotic variance involved extending the $L_{0}^{2}(\mathrm{E}, \pi)$ characterization with a specific result for this case, Lemma 2. We are not aware of general results for reversible Markov chains ensuring that ergodic averages of functions that are in $L_{0}^{1}(\mathrm{E}, \pi)$ but not $L_{0}^{2}(\mathrm{E}, \pi)$ do not have finite asymptotic variance, which would allow the characterization of Theorem 1 to be extended.

3.2. Comparison with self-normalized importance sampling. Self-normalized importance sampling is an alternative way to define a Monte Carlo approximation of $\pi(f)$ using a sequence of independent $\mu$-distributed random variables $\left(Z_{n}\right)_{n \in \mathbb{N}}$. If we define

$$
\pi_{n}^{\mathrm{SNIS}}(f):=\frac{\sum_{i=1}^{n} w\left(Z_{i}\right) f\left(Z_{i}\right)}{\sum_{i=1}^{n} w\left(Z_{i}\right)}, \quad n \in \mathbb{N},
$$

one obtains that $\sqrt{n}\left\{\pi_{n}^{\text {SNIS }}(f)-\pi(f)\right\}$ converges weakly to a $N\left(0, \pi\left(w \cdot \bar{f}^{2}\right)\right)$ random variable whenever $\pi\left(w \cdot \bar{f}^{2}\right)<\infty$, where $\bar{f}=f-\pi(f)$. Theorem 2 indicates that the class of $L_{0}^{1}(\mathrm{E}, \pi)$ functions $f$ with finite $\operatorname{var}(f, P)$ is in general smaller than those satisfying $w \cdot f \in L_{0}^{2}(\mathrm{E}, \mu)$. In particular, small values of $w$ are able to counterbalance large values of $f$ in $\pi_{n}^{\text {SNIS }}(f)$ while $\varrho \leq 1$ prevents any such counterbalancing for the IMH, as can be seen from Lemma 3. The following bounds allow us to compare $\operatorname{var}(f, P)$ with the limiting variance in the self-normalized importance sampling CLT: the former is always larger than the latter.

Proposition 2. If $f \in L_{0}^{2}(\mathrm{E}, \pi)$ and $w \cdot f \in L_{0}^{2}(\mathrm{E}, \mu)$, we have

$$
2 \pi(\varrho) \tilde{\pi}\left(f^{2} / \varrho^{2}\right)-\pi\left(f^{2}\right) \leq \operatorname{var}(f, P) \leq 2 \tilde{\pi}\left(f^{2} / \varrho^{2}\right)-\pi\left(f^{2}\right),
$$

and $\operatorname{var}(f, P) \geq \pi\left(w \cdot f^{2}\right)$.

Proof. Proposition 3 and Remark 1 of Doucet et al. (2015) show that for the IMH, $\tilde{P}$ is a positive operator on $L_{0}^{2}(\mathrm{E}, \tilde{\pi})$ so $\operatorname{var}(f / \varrho, \tilde{P}) \geq \tilde{\pi}\left(f^{2} / \varrho^{2}\right)$. Lemma 3 implies that $\operatorname{Gap}(\tilde{P}) \geq \pi(\varrho)$ and spectral considerations [see, e.g., Section 3.5 of Geyer (1992), based on Kipnis and Varadhan (1986)] give

$$
\tilde{\pi}\left(f^{2} / \varrho^{2}\right) \leq \operatorname{var}(f / \varrho, \tilde{P}) \leq \frac{2-\operatorname{Gap}(\tilde{P})}{\operatorname{Gap}(\tilde{P})} \tilde{\pi}\left(f^{2} / \varrho^{2}\right) \leq \frac{2-\pi(\varrho)}{\pi(\varrho)} \tilde{\pi}\left(f^{2} / \varrho^{2}\right) .
$$


These inequalities, together with (4), imply the first set of inequalities. The last inequality follows from Corollary 2 since

$$
2 \pi(\varrho) \tilde{\pi}\left(f^{2} / \varrho^{2}\right)-\pi\left(f^{2}\right) \geq \pi\left(w \cdot f^{2}\right)+\pi\left(f^{2} / \varrho\right)-\pi\left(f^{2}\right) \geq \pi\left(w \cdot f^{2}\right) .
$$

REMARK 4. When $f \in L_{0}^{2}(\mathrm{E}, \pi)$ and $\bar{w}=\sup _{x \in \mathrm{X}} w(x)<\infty$, spectral considerations provide the bounds $\pi\left(f^{2}\right) \leq \operatorname{var}(f, P) \leq(2 \bar{w}-1) \pi\left(f^{2}\right)$. The upper bound can be smaller or larger than the upper bound in Proposition 2, but the first lower bound of Proposition 2 is always larger than $\pi\left(f^{2}\right)$.

4. Pseudo-marginal Markov chains. We briefly motivate the construction of pseudo-marginal chains, following the notation of Andrieu and Vihola (2015). Let $\bar{\pi}$ be a probability measure on $(\mathrm{X}, \mathcal{X})$, and $\bar{X}$ the $\bar{\pi}$-reversible Metropolis-Hastings chain with proposal kernel $q$ and acceptance probability function $\bar{\alpha}(x, y):=1 \wedge$ $\bar{r}(x, y)$, where

$$
\bar{r}(x, y):=\frac{\bar{\pi}(\mathrm{d} y) \bar{q}(y, \mathrm{~d} x)}{\bar{\pi}(\mathrm{d} x) \bar{q}(x, \mathrm{~d} y)}, \quad x, y \in \mathrm{X} .
$$

Letting $\bar{\pi}$ and $\bar{q}$ have densities, also denoted by $\bar{\pi}$ and $\bar{q}$, w.r.t. some reference measure, an associated pseudo-marginal Markov chain $X$ can be constructed when only unbiased, nonnegative estimates of $\bar{\pi}(x)$ are available for each $x \in \mathrm{X}$. That is, there exists a collection of probability measures $\left\{Q_{x}: x \in \mathrm{X}\right\}$ on nonnegative noise variables such that

$$
\int_{\mathbb{R}_{+}} u Q_{x}(\mathrm{~d} u)=1, \quad x \in \mathrm{X},
$$

and so if $U \sim Q_{x}$ then $U \bar{\pi}(x)$ is a nonnegative random variable with expectation $\bar{\pi}(x)$. Defining the probability measure on $(\mathrm{E}, \mathcal{E})=\left(\mathrm{X} \times \mathbb{R}_{+}, \mathcal{X} \times \mathcal{B}\left(\mathbb{R}_{+}\right)\right)$,

$$
\pi(\mathrm{d} x, \mathrm{~d} u):=\bar{\pi}(\mathrm{d} x) Q_{x}(\mathrm{~d} u) u,
$$

the pseudo-marginal chain $X$ is a $\pi$-reversible Metropolis-Hastings chain with proposal kernel $q(x, u ; \mathrm{d} y, \mathrm{~d} v):=\bar{q}(x, \mathrm{~d} y) Q_{y}(\mathrm{~d} v)$, and acceptance probability function $\alpha(x, u ; y, v):=1 \wedge r(x, u ; y, v)$, where

$$
r(x, u ; y, v):=\bar{r}(x, y) \frac{v}{u}=\frac{v \bar{\pi}(\mathrm{d} y) \bar{q}(y, \mathrm{~d} x)}{u \bar{\pi}(\mathrm{d} x) \bar{q}(x, \mathrm{~d} y)}, \quad(x, u),(y, v) \in \mathrm{E} .
$$

From a computational perspective, this means that only variables representing the unbiased estimates $u \bar{\pi}(x)$ and $v \bar{\pi}(y)$ of the densities $\bar{\pi}(x)$ and $\bar{\pi}(y)$ are required to compute $\alpha$. Since the ratio of these densities appears in $r$, unbiased estimates of the density $\bar{\pi}$ up to a common, but unknown, normalizing constant are also sufficient; we focus here without loss of generality on the case (9) to simplify the presentation of the results, rather than allowing the right-hand side therein to be an arbitrary constant $c>0$, in which case $\bar{\pi}(\mathrm{d} x) Q_{x}(\mathrm{~d} u) u$ would define an unnormalized version of $\pi$ when $c \neq 1$. 
The influence of $\left\{Q_{x}: x \in \mathrm{X}\right\}$ on the behaviour of $X$ and associated ergodic averages has recently been the subject of intense research. For example, it is known that if the noise variables $U \sim Q_{x}$ are not almost surely bounded for $\bar{\pi}$ almost all $x$, then $X$ cannot be variance bounding, while if they are essentially uniformly bounded, then $X$ "inherits" variance bounding from $\bar{X}$ [Andrieu and Roberts (2009), Andrieu and Vihola (2015)]. In between these cases, which is fairly common in statistical applications, the situation is more complex and $X$ may or may not inherit variance bounding depending on $\bar{q}$ [see, e.g., Lee and Łatuszyński (2014), Andrieu and Vihola (2015)].

A simplified version of a result by Andrieu and Vihola (2016) establishes a partial order between different pseudo-marginal chains with noise variable distributions obtained by averaging independent realizations of each $x$-dependent noise variable a fixed number of times, thus extending results in Andrieu and Vihola (2015) on the convergence of finite asymptotic variances to their marginal counterparts in this setting. The issue of which ergodic averages have finite asymptotic variances when $X$ is not variance bounding, however, has been resolved only in a few specific settings through sub-geometric drift and minorization conditions [Andrieu and Vihola (2016), Remark 11]. In addition, a result by Bornn et al. (2017) and its generalization by Sherlock, Thiery and Lee (2017) shows that the class of functions with finite asymptotic variance cannot be enlarged by averaging in the manner just described.

The pseudo-marginal kernel $P$ described above can be written for $A \in \mathcal{E}$ as

$$
P(x, u ; A):=\int_{A} \bar{q}(x, \mathrm{~d} y) Q_{y}(\mathrm{~d} v) \alpha(x, u ; y, v)+[1-\varrho(x, u)] \mathbf{1}_{A}(x, u),
$$

where $\varrho(x, u):=\int_{\mathrm{E}} \bar{q}(x, \mathrm{~d} y) Q_{y}(\mathrm{~d} v) \alpha(x, u ; y, v)$, and the marginal kernel $\bar{P}$ can be written, for $A \in \mathcal{X}$,

$$
\bar{P}(x, A):=\int_{A} \bar{q}(x, \mathrm{~d} y) \bar{\alpha}(x, y)+[1-\bar{\varrho}(x)] \mathbf{1}_{A}(x),
$$

where $\bar{\varrho}(x):=\int_{\mathrm{E}} \bar{q}(x, \mathrm{~d} y) \bar{\alpha}(x, y)$.

Our results are most easily stated by making reference to the second moments of the noise variables, so we define

$$
s(x):=\int_{\mathbb{R}_{+}} u^{2} Q_{x}(\mathrm{~d} u), \quad x \in \mathrm{X},
$$

and $\bar{s}:=\bar{\pi}$-ess sup $s$.

4.1. Independent proposals. Our first result is a complete characterization of the functions $f \in L_{0}^{1}(\mathrm{E}, \pi)$ satisfying $\operatorname{var}(f, P)<\infty$ in the specific case where $P$ is also an IMH sampler and is essentially a corollary of Theorem 2. 
Proposition 3. Assume $\bar{q}(x, \cdot)=\bar{\mu}(\cdot)$ for all $x \in \mathrm{X}$, and $f \in L_{0}^{1}(\mathrm{E}, \pi)$. Then $\operatorname{var}(f, P)<\infty$ if and only if $f \in L_{0}^{2}(\mathrm{E}, \pi)$ and

$$
\int_{\mathrm{E}} u^{2} \frac{\mathrm{d} \bar{\pi}}{\mathrm{d} \bar{\mu}}(x) f(x, u)^{2} \bar{\pi}(\mathrm{d} x) Q_{x}(\mathrm{~d} u)<\infty .
$$

Proof. If we define $\mu(\mathrm{d} x, \mathrm{~d} u)=\bar{\mu}(\mathrm{d} x) Q_{x}(\mathrm{~d} u)$, then $P$ is exactly the $\pi$ reversible IMH kernel with proposal $\mu$ and, in particular,

$$
w(x, u)=\frac{\mathrm{d} \pi}{\mathrm{d} \mu}(x, u)=u \frac{\mathrm{d} \bar{\pi}}{\mathrm{d} \bar{\mu}}(x) .
$$

Theorem 2 then implies that $\operatorname{var}(f, P)<\infty$ if and only if $f \in L_{0}^{2}(\mathrm{E}, \pi)$ and $w \cdot f \in$ $L_{0}^{2}(\mathrm{E}, \mu)$, and so the result follows from

$$
\begin{aligned}
\mu\left(w^{2} \cdot f^{2}\right) & =\pi\left(w \cdot f^{2}\right)=\int_{\mathrm{E}} u \frac{\mathrm{d} \bar{\pi}}{\mathrm{d} \bar{\mu}}(x) f(x, u)^{2} \pi(\mathrm{d} x, \mathrm{~d} u) \\
& =\int_{\mathrm{E}} u^{2} \frac{\mathrm{d} \bar{\pi}}{\mathrm{d} \bar{\mu}}(x) f(x, u)^{2} \bar{\tau}(\mathrm{d} x) Q_{x}(\mathrm{~d} u) .
\end{aligned}
$$

Corollary 3. Assume $\bar{q}(x, \cdot)=\bar{\mu}(\cdot)$ for all $x \in \mathrm{X}$. If $f(\cdot, u)=f_{X} \in$ $L_{0}^{2}(\mathrm{X}, \bar{\pi})$, then $\operatorname{var}(f, P)<\infty$ if and only if

$$
\int_{\mathrm{X}} s(x) \frac{\mathrm{d} \bar{\pi}}{\mathrm{d} \bar{\mu}}(x) f_{X}(x)^{2} \bar{\pi}(\mathrm{d} x)<\infty,
$$

which is clearly satisfied if $\bar{s}<\infty$ and $\bar{\pi}$-ess sup $\mathrm{d} \bar{\pi} / \mathrm{d} \bar{\mu}<\infty$.

REMARK 5. It is possible that $\sup _{x \in \mathrm{X}} s(x) \frac{\mathrm{d} \bar{\pi}}{\mathrm{d} \bar{\mu}}(x)<\infty$ even though $\bar{s}=\infty$. Let, for example, $\bar{\pi}(\mathrm{d} x) \propto h(x) p(\mathrm{~d} x)$ and $\bar{\mu}=p$, where $h: \mathrm{X} \rightarrow(0,1), p$ is a probability measure and $Q_{x}(\{1 / h(x)\})=h(x)=1-Q_{x}(\{0\})$. Then one obtains $s(x)=h(x)^{-1}$ and $\frac{\mathrm{d} \bar{\tau}}{\mathrm{d} \bar{\mu}}(x) \propto h(x)$, so that $s(x) \frac{\mathrm{d} \bar{\tau}}{\mathrm{d} \bar{\mu}}(x)$ is constant for all $x \in \mathrm{X}$. This is equivalent to the IMH for a simple approximate Bayesian computation model, where $p$ is the prior distribution of the statistical parameter and $h(x)$ the probability of the observed data when $x$ is the true parameter [Tavaré et al. (1997)].

REMARK 6. If for some $C>0, C^{-1} \leq \frac{\mathrm{d} \bar{\pi}}{\mathrm{d} \bar{\mu}}(x) \leq C$ for all $x \in \mathrm{X}$, then $\bar{s}<\infty$ is both necessary and sufficient for all ergodic averages of functions in $L_{0}^{2}(\mathrm{X}, \bar{\pi})$ to have finite asymptotic variance. Perhaps surprisingly, using $\bar{\pi}$ as the proposal distribution can make the class of functions with finite asymptotic variance smaller when $\bar{s}=\infty$ : in the example of Remark 5 , we obtain that this class is exactly $L_{0}^{2}(\mathrm{X}, p)$. Under this same condition, we also observe that a necessary and sufficient condition for all bounded functions $f_{X}$ to have finite asymptotic variance is $\int_{\mathrm{X}} s(x) \bar{\pi}(\mathrm{d} x)<\infty$. 
That the class of functions whose ergodic averages have finite asymptotic variance depends on the second moment function $s$ is entirely consistent with results by Bornn et al. (2017) and Sherlock, Thiery and Lee (2017). In particular, let us define for $N \in \mathbb{N}$ a new collection of induced probability measures $\left\{Q_{x}^{N}: x \in \mathrm{X}\right\}$, where for each $x \in \mathrm{X}, U \sim Q_{x}^{N}$ is equal in distribution to the average of $N$ independent, $Q_{x}$-distributed random variables. Letting $v(x)$ be the variance of $U \sim Q_{x}$, we obtain $s(x)=1+v(x)$ whereas the second moment function $s_{N}$ associated with $\left\{Q_{x}^{N}: x \in \mathrm{X}\right\}$ satisfies $s_{N}(x)=1+v(x) / N$. It is then clear that

$$
\int_{\mathrm{X}} s(x) \frac{\mathrm{d} \bar{\pi}}{\mathrm{d} \bar{\mu}}(x) f_{X}(x)^{2} \bar{\pi}(\mathrm{d} x)<\infty \quad \Longleftrightarrow \quad \int_{\mathrm{X}} s_{N}(x) \frac{\mathrm{d} \bar{\pi}}{\mathrm{d} \bar{\mu}}(x) f_{X}(x)^{2} \overline{\bar{\pi}}(\mathrm{d} x)<\infty .
$$

4.2. An auxiliary Markov kernel. The remainder of our results provide sufficient conditions for the ergodic averages of a function in $L^{2}(E, \pi)$ to have finite asymptotic variance. The proofs are based on a modification of $P$ whose associated asymptotic variances are larger than or equal to those associated with $P$ itself, so that the novel converse part of Theorem 1 can still be applied to obtain results. Strictly for the purposes of this analysis, as in Andrieu and Vihola (2015) and Doucet et al. (2015), we introduce an auxiliary Markov kernel $R$ that has the same proposal as $P$ but a different acceptance probability function. In particular, the acceptance probability is

$$
\alpha_{R}(x, u ; y, v):=\{1 \wedge \bar{r}(x, y)\}\left\{1 \wedge \frac{v}{u}\right\} .
$$

We can therefore write $R$ as

$$
R(x, u ; A):=\int_{A} \bar{q}(x, \mathrm{~d} y) Q_{y}(\mathrm{~d} v) \bar{\alpha}(x, y)\left\{1 \wedge \frac{v}{u}\right\}+\left[1-\varrho_{R}(x, u)\right] \mathbf{1}_{A}(x, u),
$$

where

$$
\varrho_{R}(x, u):=\int_{\mathrm{E}} \bar{q}(x, \mathrm{~d} y) Q_{y}(\mathrm{~d} v) \alpha_{R}(x, u ; y, v) .
$$

It is straightforward to deduce that $R$ is $\pi$-reversible, for example, by Lemma 2 of Banterle et al. (2015), and also that $\alpha_{R}(x, u ; y, v) \leq \alpha(x, u ; y, v)$ for all $(x, u),(y, v) \in \mathrm{E}$. The kernels $P$ and $R$ are therefore ordered in the sense of Peskun [Peskun (1973), Tierney (1998)], so $\operatorname{var}(f, P) \leq \operatorname{var}(f, R)$ for all $f \in L^{2}(\mathrm{E}, \pi)$.

Lemma 5 below could be deduced from Proposition 8 of Andrieu and Vihola (2015), in which the context is slightly different; we provide a proof for completeness. This result allows us to compare the spectral gaps of closely related Markov kernels that operate on different spaces, and is central to our analysis of pseudomarginal Markov chains.

LEMMA 5. Let $\mu(\mathrm{d} x, \mathrm{~d} u)=v(\mathrm{~d} x) \mu_{x}(\mathrm{~d} u)$ be a measure on $(\mathrm{E}, \mathcal{E})=$ $(\mathrm{X} \times \mathrm{U}, \mathcal{X} \times \mathcal{U})$. Let $Q$ be a v-reversible sub-Markov kernel on $(\mathrm{X}, \mathcal{X}), \varrho$ be 
the function $x \mapsto Q(x, \mathrm{X})$ and $\bar{P}$ be the $v$-reversible Markov kernel

$$
\bar{P}(x, A)=\int_{A} Q(x, \mathrm{~d} y)+[1-\varrho(x)] \mathbf{1}_{A}(x), \quad A \in \mathcal{X} .
$$

Letting $P$ be the $\mu$-reversible kernel

$$
P(x, u ; A)=\int_{A} Q(x, \mathrm{~d} y) \mu_{y}(\mathrm{~d} v)+[1-\varrho(x)] \mathbf{1}_{A}(x, u), \quad A \in \mathcal{E},
$$

we have $\operatorname{Gap}(\bar{P}) \wedge \varrho^{*} \leq \operatorname{Gap}(P) \leq \operatorname{Gap}(\bar{P})$, where $\varrho^{*}=v$ - essinf $\varrho$.

Proof. Let $f \in L_{0}^{2}(\mathrm{E}, \mu)$ with $\langle f, f\rangle_{\mu}=1$. For each $x \in \mathrm{X}$, we write $f_{x}$ for the function $u \mapsto f(x, u)$. Let $\bar{f}(x):=\mu_{x}\left(f_{x}\right)=\int_{\mathrm{U}} f(x, u) \mu_{x}(\mathrm{~d} u)$ and note that $\bar{f} \in L_{0}^{2}(\mathrm{X}, v)$. When a function $g \in L^{2}(\mathrm{X}, v)$ is treated as a function in $L^{2}(\mathrm{E}, \mu)$, we adopt the convention that $g(\cdot, u)=g$. We observe that

$$
\mathcal{E}_{P}(f)=\langle\varrho \cdot f, f\rangle_{\mu}-\langle\varrho \cdot \bar{f}, \bar{f}\rangle_{\nu}+\mathcal{E}_{\bar{P}}(\bar{f}) .
$$

Let $h(x)=\operatorname{var}_{\mu_{x}}\left(f_{x}\right)$. Then for any $g \in L^{2}(\mathrm{X}, v)$ we have $\langle g \cdot f, f\rangle_{\mu}-\langle g$. $\bar{f}, \bar{f}\rangle_{\nu}=\langle g, h\rangle_{\nu}$, and so

$$
\begin{aligned}
\mathcal{E}_{P}(f) & =\langle\varrho, h\rangle_{\nu}+\mathcal{E}_{\bar{P}}(\bar{f}) \geq\langle\varrho, h\rangle_{\nu}+\langle\bar{f}, \bar{f}\rangle_{\nu} \operatorname{Gap}(\bar{P}) \\
& \geq\langle\varrho, h\rangle_{\nu}+\langle\bar{f}, \bar{f}\rangle_{\nu} \operatorname{Gap}(\bar{P}) \wedge \varrho^{*} \\
& =\langle\varrho, h\rangle_{\nu}+\left\{\langle f, f\rangle_{\mu}-\langle 1, h\rangle_{\nu}\right\} \operatorname{Gap}(\bar{P}) \wedge \varrho^{*} \\
& =\operatorname{Gap}(\bar{P}) \wedge \varrho^{*}+\langle\varrho, h\rangle_{\nu}-\left\langle\operatorname{Gap}(\bar{P}) \wedge \varrho^{*}, h\right\rangle_{\nu} \\
& \geq \operatorname{Gap}(\bar{P}) \wedge \varrho^{*} .
\end{aligned}
$$

Since $f \in L_{0}^{2}(\mathrm{E}, \mu)$ is arbitrary with $\langle f, f\rangle_{\mu}=1$, we obtain from (2) that $\operatorname{Gap}(P) \geq \operatorname{Gap}(\bar{P}) \wedge \varrho^{*}$. That $\operatorname{Gap}(P) \leq \operatorname{Gap}(\bar{P})$ also follows from (2) by considering functions $f$ of $x$ alone in $L_{0}^{2}(\mathrm{E}, \mu)$, since then $\mathcal{E}_{P}(f)=\mathcal{E}_{\bar{P}}(\bar{f})$.

4.3. Independent noise distributions. Our first result assumes that the noise distribution is state-independent, that is, $Q_{x}=Q$ for all $x \in \mathrm{X}$, and that the marginal jump chain is variance bounding.

Proposition 4. Assume that $Q_{x}=Q$ for all $x \in \mathrm{X}, \bar{s}<\infty$ and that the jump kernel associated with $\bar{P}$ is variance bounding. Then:

1. For $f \in L_{0}^{2}(\mathrm{E}, \pi), \operatorname{var}(f, P)<\infty$ if

$$
\int_{\mathrm{E}}(u+\bar{s}) u \frac{f(x, u)^{2}}{\bar{\varrho}(x)} \bar{\pi}(\mathrm{d} x) Q(\mathrm{~d} u)<\infty .
$$

2. If $f(\cdot, u)=f_{X} \in L_{0}^{2}(\mathrm{X}, \bar{\pi})$, then $\operatorname{var}\left(f_{X}, \bar{P}\right)<\infty \Rightarrow \operatorname{var}(f, P)<\infty$. 
These results complement the results of Doucet et al. (2015) and Sherlock et al. (2015), who assume that the distribution of the weights is independent of $x$ in order to optimize the trade-off between computational cost and asymptotic variance. In particular, Proposition 4 indicates that those results can be applied to ergodic averages of all $L_{0}^{2}(\mathrm{X}, \bar{\pi})$ functions when the jump kernel associated with $\bar{P}$ is variance bounding.

When the noise distribution is state-independent, (10) simplifies to

$$
R(x, u ; A)=\int_{A} \bar{q}(x, \mathrm{~d} y) Q(\mathrm{~d} v)\{1 \wedge r(x, y)\}\left\{1 \wedge \frac{v}{u}\right\}+\left[1-\varrho_{R}(x, u)\right] \mathbf{1}_{A}(x, u),
$$

where $\varrho_{R}(x, u)=\int_{\mathrm{E}} \bar{q}(x, \mathrm{~d} y) Q(\mathrm{~d} v) \alpha_{R}(x, u ; y, v)$. If we define

$$
\varrho_{U}(u):=\int_{\mathbb{R}_{+}} Q(\mathrm{~d} v)\left\{1 \wedge \frac{v}{u}\right\}, \quad u \in \mathbb{R}_{+},
$$

then we observe that $\varrho_{R}(x, u)=\bar{\varrho}(x) \varrho_{U}(u)$.

LEMMA 6. With $\bar{s}=\int_{\mathbb{R}_{+}} u^{2} Q(\mathrm{~d} u), \varrho_{U}$ in (12) satisfies

$$
\frac{1}{\bar{s}+u} \leq \varrho_{U}(u) \leq 1 \wedge \frac{1}{u},
$$

and $\int_{\mathbb{R}_{+}} Q(\mathrm{~d} u) u \varrho_{U}(u) \geq(2 \bar{s})^{-1}$.

PROOF. The first part follows from Lemma 1 , since $\varrho_{U}(u)=\mathbb{E}\left[1 \wedge \frac{V}{u}\right]$, where $V \sim Q$, and $V$ is a nonnegative random variable with expectation 1 . The second part follows from the first part and Jensen's inequality.

ProOf OF Proposition 4. For clarity, denote by $P^{*}$ the jump kernel associated with $\bar{P}$ and let $\tilde{R}$ be the jump Markov kernel associated with $R$, that is,

$$
\tilde{R}(x, u ; \mathrm{d} y, \mathrm{~d} v):=\frac{\bar{q}(x, \mathrm{~d} y) Q(\mathrm{~d} v)\{1 \wedge \bar{r}(x, y)\}\left\{1 \wedge \frac{v}{u}\right\}}{\varrho_{R}(x, u)} .
$$

From (3) and $\varrho_{R}(x, u)=\bar{\varrho}(x) \varrho_{U}(u), \tilde{R}$ is $\tilde{\pi}$-reversible where, with Id the identity function,

$$
\tilde{\pi}(\mathrm{d} x, \mathrm{~d} u):=\frac{\bar{\pi}(\mathrm{d} x) \bar{\varrho}(x)}{\bar{\pi}(\bar{\varrho})} \frac{Q(\mathrm{~d} u) u \varrho_{U}(u)}{Q\left(\operatorname{Id} \cdot \varrho_{U}\right)} .
$$

The strategy of the proof is to deduce that $\operatorname{Gap}(\tilde{R})>0$ from the fact that $\operatorname{Gap}\left(P^{*}\right)>0$. We then identify which functions $f$ satisfy $f / \varrho_{R} \in L_{0}^{2}(\mathrm{E}, \mu)$, since then $\operatorname{var}(f, P) \leq \operatorname{var}(f, R)<\infty$. Since $P^{*}$ and $\tilde{R}$ operate on different spaces, $\mathrm{X}$ and $\mathrm{E}=\mathrm{X} \times \mathrm{U}$, respectively, in order to compare their spectral gaps we will apply 
Lemma 5. We cannot do this directly, and thus we will introduce an auxiliary kernel $M$, such that the right gaps of $M$ and $P^{*}$ can be compared using Lemma 5 and such that

$$
\operatorname{Gap}(M)>0 \Rightarrow \operatorname{Gap}(\tilde{R})>0 .
$$

Notice that we can express $\tilde{R}$ as

$$
\begin{aligned}
\tilde{R}(x, u ; \mathrm{d} y, \mathrm{~d} v) & =\frac{\bar{q}(x, \mathrm{~d} y)\{1 \wedge \bar{r}(x, y)\} Q(\mathrm{~d} v)\left\{1 \wedge \frac{v}{u}\right\}}{\bar{\varrho}(x) \varrho U(u)} \\
& =P^{*}(x, \mathrm{~d} y) \frac{Q(\mathrm{~d} v)\left\{1 \wedge \frac{v}{u}\right\}}{\varrho_{U}(u)} .
\end{aligned}
$$

We introduce the $\tilde{\pi}$-reversible auxiliary Markov kernel $M$ by modifying $\tilde{R}$ so that the second coordinate forms a sequence of independent draws from the marginal under $\tilde{\pi}$

$$
\begin{aligned}
M(x, u ; \mathrm{d} y, \mathrm{~d} v) & :=\frac{\bar{q}(x, \mathrm{~d} y)\{1 \wedge \bar{r}(x, y)\}}{\bar{\varrho}(x)} \frac{Q(\mathrm{~d} v) v \varrho_{U}(v)}{Q\left(\mathrm{Id} \cdot \varrho_{U}\right)} \\
& =P^{*}(x, \mathrm{~d} y) \frac{Q(\mathrm{~d} v) v \varrho_{U}(v)}{Q\left(\mathrm{Id} \cdot \varrho_{U}\right)}
\end{aligned}
$$

This choice allows to immediately deduce $\operatorname{Gap}\left(P^{*}\right)>0 \Rightarrow \operatorname{Gap}(M)>0$ by applying Lemma 5 with $Q=P^{*}$.

We will now compare $\operatorname{Gap}(M)$ and $\operatorname{Gap}(\tilde{R})$. Notice that

$$
\begin{aligned}
\tilde{R}(x, u ; \mathrm{d} y, \mathrm{~d} v) & =\frac{\bar{q}(x, \mathrm{~d} y)\{1 \wedge \bar{r}(x, y)\}}{\bar{\varrho}(x)} \frac{Q(\mathrm{~d} v) v\left\{\frac{1}{v} \wedge \frac{1}{u}\right\}}{\varrho_{U}(u)} \\
& =M(x, u ; \mathrm{d} y, \mathrm{~d} v) \frac{1}{\varrho_{U}(u) \varrho_{U}(v)[v \vee u]} Q\left(\mathrm{Id} \cdot \varrho_{U}\right) .
\end{aligned}
$$

From Lemma 6, we have $\varrho_{U}(u) \leq 1 \wedge 1 / u$, so that $\varrho_{U}(u) \varrho_{U}(v)[v \vee u] \leq 1$ by the same argument as in the proof of Lemma 3. Hence, $\tilde{R}(x, u ; \mathrm{d} y, \mathrm{~d} v) \geq Q$ (Id . $\left.\varrho_{U}\right) M(x, u ; \mathrm{d} y, \mathrm{~d} v)$, and it follows that

$$
0<\mathcal{E}_{M}(f) \leq Q\left(\mathrm{Id} \cdot \varrho_{U}\right)^{-1} \mathcal{E}_{\tilde{R}}(f), \quad f \in L_{0}^{2}(\mathrm{E}, \mu)
$$

and so $\operatorname{Gap}(\tilde{R}) \geq Q\left(\mathrm{Id} \cdot \varrho_{U}\right) \operatorname{Gap}(M)>0$ since $Q\left(\mathrm{Id} \cdot \varrho_{U}\right) \geq(2 \bar{s})^{-1}$ by Lemma 6 . Since $\operatorname{var}(f, R) \geq \operatorname{var}(f, P)$, an application of Corollary 1 shows that all $f \in$ $L_{0}^{2}(\mathrm{E}, \pi)$ such that $f / \varrho_{R} \in L_{0}^{2}(\mathrm{E}, \mu)$ satisfy $\operatorname{var}(f, P)<\infty$ and we conclude the first part by writing

$$
\begin{aligned}
\pi\left(\varrho_{R}\right) \mu\left(f^{2} / \varrho_{R}^{2}\right) & =\int_{\mathrm{E}} \frac{f(x, u)^{2}}{\varrho_{R}(x, u)} \pi(\mathrm{d} x, \mathrm{~d} u)=\int_{\mathrm{E}} \frac{f(x, u)^{2}}{\bar{\varrho}(x) \varrho_{U}(u)} \bar{\pi}(\mathrm{d} x) Q(\mathrm{~d} u) u \\
& \leq \int_{\mathrm{E}}(u+\bar{s}) u \frac{f(x, u)^{2}}{\bar{\varrho}(x)} \bar{\pi}(\mathrm{d} x) Q(\mathrm{~d} u),
\end{aligned}
$$


where the inequality follows from Lemma 6. For the second part, we have

$$
\begin{aligned}
\pi\left(\varrho_{R}\right) \mu\left(f^{2} / \varrho_{R}^{2}\right) & \leq \int_{\mathrm{X}} \frac{f_{X}(x)^{2}}{\bar{\varrho}(x)} \bar{\pi}(\mathrm{d} x) \int_{\mathbb{R}^{+}}(u+\bar{s}) u Q(\mathrm{~d} u) \\
& \leq 2 \bar{s} \int_{\mathrm{X}} \frac{f_{X}(x)^{2}}{\bar{\varrho}(x)} \bar{\pi}(\mathrm{d} x),
\end{aligned}
$$

and $\bar{\pi}\left(f_{X}^{2} / \bar{\varrho}\right)<\infty$ is equivalent by Corollary 1 to $\operatorname{var}\left(f_{X}, \bar{P}\right)<\infty$ since $P^{*}$ is variance bounding.

4.4. General case. Our most generally applicable result for pseudo-marginal chains is the following. The strategy of the proof is similar in many respects to that of Proposition 4 , but more complicated. In addition, the assumption that $\bar{P}$ is variance bounding is stronger (cf. Proposition 1) than the assumption that its associated jump kernel is variance bounding.

THEOREM 3. Assume $\bar{P}$ is variance bounding and $\bar{s}<\infty$. Then for $f \in$ $L_{0}^{2}(\mathrm{E}, \pi)$ satisfying

$$
\int_{\mathrm{E}} f(x, u)^{2} \bar{\pi}(\mathrm{d} x) Q_{x}(\mathrm{~d} u) u^{2}<\infty
$$

$\operatorname{var}(f, P)<\infty$. In particular, if $f(\cdot, u)=f_{X} \in L_{0}^{2}(\mathrm{X}, \bar{\pi})$ then $\operatorname{var}(f, P)<\infty$.

Remark 6 indicates that the condition $\bar{s}<\infty$ is also necessary in some settings, while of course Remark 5 indicates that it is not necessary in others.

In this case, $\varrho_{R}$ does not factorize as in Section 4.3 since the distribution of the weights is dependent on the proposed value of $y$. In order to define appropriate auxiliary kernels to allow the application of Lemma 5 in the proof of Theorem 3, we define the expected acceptance probability associated to a point $x \in \mathrm{X}$ as

$$
\varrho_{R, X}(x):=\int Q_{x}(\mathrm{~d} u) u \varrho_{R}(x, u) .
$$

LEMMA 7. Let $\varrho_{R}$ and $\varrho_{R, X}$ be given by (11) and (14), respectively. Then for $\operatorname{each}(x, u) \in \mathrm{E}$,

$$
\frac{\bar{\varrho}(x)}{\bar{s}+u} \leq \int_{\mathrm{X}} \bar{q}(x, \mathrm{~d} y) \bar{\alpha}(x, y) \frac{\bar{\varrho}(x)}{s(y)+u} \leq \varrho_{R}(x, u) \leq \bar{\varrho}(x)\left\{1 \wedge \frac{1}{u}\right\},
$$

and for each $x \in \mathrm{X}, \bar{\varrho}(x) /(2 \bar{s}) \leq \varrho_{R, X}(x) \leq \bar{\varrho}(x)$.

PROOF. We can write $\varrho_{R}(x, u)=\int_{\mathrm{X}} \bar{q}(x, \mathrm{~d} y) \bar{\alpha}(x, y) \int_{\mathbb{R}_{+}} Q_{y}(\mathrm{~d} v)\left[1 \wedge \frac{v}{u}\right]$, whence the first part holds by applying Lemma 1 to the inner integral. For the 
second part, we have

$$
\begin{aligned}
\varrho_{R, X}(x) & =\int_{\mathbb{R}_{+}} Q_{x}(\mathrm{~d} u) u \varrho_{R}(x, u) \\
& =\int_{\mathrm{X}} \bar{q}(x, \mathrm{~d} y) \bar{\alpha}(x, y) \int_{\mathbb{R}_{+}^{2}} Q_{x}(\mathrm{~d} u) u Q_{y}(\mathrm{~d} v)\left[1 \wedge \frac{v}{u}\right],
\end{aligned}
$$

so that $\varrho_{R, X}(x) \leq \bar{\varrho}(x)$. Moreover,

$$
\begin{aligned}
\varrho_{R, X}(x) & =\int_{\mathrm{X}} \bar{q}(x, \mathrm{~d} y) \bar{\alpha}(x, y) \int_{\mathbb{R}_{+}^{2}} Q_{x}(\mathrm{~d} u) u Q_{y}(\mathrm{~d} v) v\left[\frac{1}{v} \wedge \frac{1}{u}\right] \\
& \geq \int_{\mathrm{X}} \bar{q}(x, \mathrm{~d} y) \bar{\alpha}(x, y) /[s(x)+s(y)] \geq \int_{\mathrm{X}} \bar{q}(x, \mathrm{~d} y) \bar{\alpha}(x, y) /(2 \bar{s}),
\end{aligned}
$$

where we have used Jensen's inequality and the fact that $a \vee b \leq a+b$.

Proof of TheOREM 3. Let $\tilde{R}$ be the jump kernel associated with $R$, which from (3) is $\mu$-reversible with

$$
\mu(\mathrm{d} x, \mathrm{~d} u)=\frac{\bar{\pi}(\mathrm{d} x) Q_{x}(\mathrm{~d} u) u \varrho_{R}(x, u)}{\pi\left(\varrho_{R}\right)} .
$$

The strategy of the proof is as follows. We will use the fact that $\bar{P}$ is variance bounding to show that $\operatorname{Gap}(\tilde{R})>0$; we will then identify which functions $f$ satisfy $f / \varrho_{R} \in L_{0}^{2}(\mathrm{E}, \mu)$, since then $\operatorname{var}(f, P) \leq \operatorname{var}(f, R)<\infty$. Since $\bar{P}$ and $\tilde{R}$ operate on $\mathrm{X}$ and $\mathrm{E}=\mathrm{X} \times \mathrm{U}$, respectively, in order to compare their spectral gaps we will apply Lemma 5 . We cannot do this directly, and thus we will introduce two auxiliary kernels, $M$ and $\bar{M}$, which do have the required structure and such that the following sequence of implications holds:

(15) $\operatorname{Gap}(\bar{P})>0 \Rightarrow \operatorname{Gap}(\bar{M})>0 \Rightarrow \operatorname{Gap}(M)>0 \Rightarrow \operatorname{Gap}(\tilde{R})>0$.

We decompose $\mu$ as $\mu(\mathrm{d} x, \mathrm{~d} u)=v(\mathrm{~d} x) \mu_{x}(\mathrm{~d} u)$ where $v(\mathrm{~d} x):=\bar{\pi}(\mathrm{d} x) \varrho_{R, X}(x) /$ $\pi\left(\varrho_{R}\right)$ and $\mu_{x}(\mathrm{~d} u):=Q_{x}(\mathrm{~d} u) u \varrho_{R}(x, u) \varrho_{R, X}(x)$. Since the $v$-reversible kernel $\bar{M}$ must inherit a right spectral gap from $\bar{P}$, we choose $\bar{M}$ to be the MetropolisHastings kernel with proposal $\bar{P}$ and target $v$, that is,

$$
\bar{M}(x, A):=\int_{A} \bar{q}(x, \mathrm{~d} y) \bar{\alpha}(x, y)\left[1 \wedge \frac{\varrho_{R, X}(y)}{\varrho_{R, X}(x)}\right]+\left[1-\varrho_{M}(x)\right] \mathbf{1}_{A}(x), \quad A \in \mathcal{X},
$$

where $\varrho_{M}(x):=\int_{\mathrm{X}} \bar{q}(x, \mathrm{~d} y) \bar{\alpha}(x, y)\left[1 \wedge \varrho_{R, X}(y) / \varrho_{R, X}(x)\right]$. The $\mu$-reversible Markov kernel $M$ is then dictated by Lemma 5 to be

$$
M(x, u ; A):=\int_{A} \bar{q}(x, \mathrm{~d} y) \bar{\alpha}(x, y)\left[1 \wedge \frac{\varrho_{R, X}(y)}{\varrho_{R, X}(x)}\right] \mu_{y}(\mathrm{~d} v)+\left[1-\varrho_{M}(x)\right] \mathbf{1}_{A}(x, u)
$$

for $A \in \mathcal{E}$, and we proceed to prove (15). 
We observe that $\bar{P}$ being variance bounding implies $\varrho:=\inf _{x} \bar{\varrho}(x)>0$, by Theorem 1 of Lee and Łatuszyński (2014). By Lemma 7, we have $\varrho_{R, X}(y) / \varrho_{R, X}(x) \geq$ $\underline{\varrho} /(2 \bar{s})$ so $\inf _{x \in \mathrm{X}} \varrho_{M}(x) \geq 1 \wedge \underline{\varrho} /(2 \bar{s})>0$. By $\operatorname{Lemma} 5, \operatorname{Gap}(M) \geq \operatorname{Gap}(\bar{M}) \wedge 1 \wedge$ $[\varrho /(2 \bar{s})]=\operatorname{Gap}(\bar{M}) \wedge[\varrho /(2 \bar{s})]$ and we now show that $\operatorname{Gap}(\bar{M})>0$. Since $\varrho>0$, $\overline{L^{2}}(\mathrm{X}, v)=L^{2}(\mathrm{X}, \bar{\pi})$. For $f \in L^{2}(\mathrm{X}, \bar{\pi})$, we have

$$
\begin{aligned}
2 \mathcal{E}_{\bar{M}}(f) & =\int_{\mathrm{X}} \frac{\bar{\pi}(\mathrm{d} x) \varrho_{R, X}(x)}{\pi\left(\varrho_{R}\right)} \bar{q}(x, \mathrm{~d} y) \bar{\alpha}(x, y)\left[1 \wedge \frac{\varrho_{R, X}(y)}{\varrho_{R, X}(x)}\right][f(y)-f(x)]^{2} \\
& \geq \frac{\varrho}{2 \bar{s}} \int \bar{\pi}(\mathrm{d} x) \bar{q}(x, \mathrm{~d} y) \bar{\alpha}(x, y)[f(y)-f(x)]^{2}=2 \frac{\varrho}{2 \bar{s}} \mathcal{E}_{\bar{P}}(f) .
\end{aligned}
$$

Moreover, for $f \in L_{0}^{2}(\mathrm{X}, \bar{\pi})$,

$$
\frac{\operatorname{var}_{\bar{\pi}}(f)}{\operatorname{var}_{v}(f)}=\frac{\pi\left(\varrho_{R}\right) \bar{\pi}\left(f^{2}\right)}{\bar{\pi}\left(\varrho_{R, X} \cdot f^{2}\right)-\bar{\pi}\left(\varrho_{R, X} \cdot f\right)^{2} / \pi\left(\varrho_{R}\right)} \geq \frac{\pi\left(\varrho_{R}\right) \bar{\pi}\left(f^{2}\right)}{\bar{\pi}\left(\varrho_{R, X} \cdot f^{2}\right)} \geq \pi\left(\varrho_{R}\right),
$$

so that for all $f \in L^{2}(\mathrm{X}, v)=L^{2}(\mathrm{X}, \bar{\pi})$,

$$
\frac{\mathcal{E}_{\bar{M}}(f)}{\operatorname{var}_{v}(f)} \geq \frac{\varrho}{2 \bar{s}} \frac{\mathcal{E}_{\bar{P}}(f)}{\operatorname{var}_{\bar{\pi}}(f)} \cdot \frac{\operatorname{var}_{\bar{\pi}}(f)}{\operatorname{var}_{\nu}(f)} \geq \frac{\varrho \pi\left(\varrho_{R}\right)}{2 \bar{s}} \frac{\mathcal{E}_{\bar{P}}(f)}{\operatorname{var}_{\bar{\pi}}(f)},
$$

and it follows from (2) that $\operatorname{Gap}(\bar{M}) \geq \operatorname{Gap}(\bar{P}) \varrho \pi\left(\varrho_{R}\right) /(2 \bar{s})>0$, and so $\operatorname{Gap}(M)>0$. Finally, we compare $\tilde{R}$ with $M$. For $f \in L_{0}^{2}(\mathrm{E}, \mu)$, we have

$$
\begin{aligned}
2 \mathcal{E}_{\tilde{R}}(f) & =\int \mu(\mathrm{d} x, \mathrm{~d} u) \frac{\bar{q}(x, \mathrm{~d} y) Q_{y}(\mathrm{~d} v) \bar{\alpha}(x, y)\left[1 \wedge \frac{v}{u}\right]}{\varrho_{R}(x, u)}[f(y, v)-f(x, u)]^{2} \\
& =\int \mu(\mathrm{d} x, \mathrm{~d} u) \frac{\bar{q}(x, \mathrm{~d} y) Q_{y}(\mathrm{~d} v) v \bar{\alpha}(x, y)\left[\frac{1}{v} \wedge \frac{1}{u}\right]}{\varrho_{R}(x, u)}[f(y, v)-f(x, u)]^{2} \\
& =\int \mu(\mathrm{d} x, \mathrm{~d} u) \frac{\bar{q}(x, \mathrm{~d} y) \bar{\alpha}(x, y)\left[\frac{1}{v} \wedge \frac{1}{u}\right]}{\varrho_{R}(x, u)} \frac{\mu_{y}(\mathrm{~d} v) \varrho_{R}, X(y)}{\varrho_{R}(y, v)}[f(y, v)-f(x, u)]^{2} .
\end{aligned}
$$

From Lemma 7 , we know that $[v \vee u] \varrho_{R}(x, u) \varrho_{R}(y, v) \leq \bar{\varrho}(x) \bar{\varrho}(y)$ for all $(x, u),(y, v) \in \mathrm{E}$, so

$$
\begin{aligned}
2 \mathcal{E}_{\tilde{R}}(f) & \geq \int \mu(\mathrm{d} x, \mathrm{~d} u) \bar{q}(x, \mathrm{~d} y) \bar{\alpha}(x, y) \mu_{y}(\mathrm{~d} v) \frac{\varrho_{R, X}(y)}{\bar{\varrho}(x) \bar{\varrho}(y)}[f(y, v)-f(x, u)]^{2} \\
& \geq \frac{1}{2 \bar{s}} \int \mu(\mathrm{d} x, \mathrm{~d} u) \bar{q}(x, \mathrm{~d} y) \bar{\alpha}(x, y) \mu_{y}(\mathrm{~d} v) \bar{\varrho}(x)^{-1}[f(y, v)-f(x, u)]^{2} \\
& \geq \frac{1}{2 \bar{s}} \int \mu(\mathrm{d} x, \mathrm{~d} u) \bar{q}(x, \mathrm{~d} y) \bar{\alpha}(x, y) \mu_{y}(\mathrm{~d} v)\left[1 \wedge \frac{\varrho_{R, X}(y)}{\varrho_{R, X}(x)}\right][f(y, v)-f(x, u)]^{2} \\
& =\frac{1}{2 \bar{s}} 2 \mathcal{E}_{M}(f) .
\end{aligned}
$$


Hence $\operatorname{Gap}(\tilde{R}) \geq \operatorname{Gap}(M) /(2 \bar{s})>0$, so by Corollary 1 all functions $f \in$ $L_{0}^{2}(\mathrm{E}, \pi)$ satisfying $f / \varrho_{R} \in L_{0}^{2}(\mathrm{E}, \mu)$ have $\operatorname{var}(f, R)<\infty$. We have

$$
\begin{aligned}
\pi\left(\varrho_{R}\right) \int_{\mathrm{E}} \frac{f(x, u)^{2}}{\varrho_{R}(x, u)^{2}} \mu(\mathrm{d} x, \mathrm{~d} u) & =\int_{\mathrm{E}} \frac{f(x, u)^{2}}{\varrho_{R}(x, u)} \bar{\pi}(\mathrm{d} x) Q_{x}(\mathrm{~d} u) u \\
& \leq \int_{\mathrm{E}} f(x, u)^{2} \frac{\bar{s}+u}{\bar{\varrho}(x)} \bar{\pi}(\mathrm{d} x) Q_{x}(\mathrm{~d} u) u \\
& \leq \frac{\bar{s}}{\underline{\varrho}} \pi\left(f^{2}\right)+\frac{1}{\underline{\varrho}} \int_{\mathrm{E}} f(x, u)^{2} u^{2} \bar{\pi}(\mathrm{d} x) Q_{x}(\mathrm{~d} u),
\end{aligned}
$$

and we conclude by noting that for $f \in L_{0}^{2}(\mathrm{E}, \pi), \operatorname{var}(f, P) \leq \operatorname{var}(f, R)$.

5. On alternatives to geometric random variables. One of the contributions of Douc and Robert (2011) is to consider weighted ergodic averages associated with the Markov chain $\tilde{X}$ to estimate $\pi(f)$. In particular, they propose alternative random weights to $\left(\tau_{n}\right)_{n \in \mathbb{N}}$ that lead to estimators of $\pi(f)$ with a smaller limiting variance in the corresponding CLT. The purpose of this last section is to point out that in many situations, the reduction in variance can be limited.

We consider the sequence of estimators of $\pi(f)$, with $\tilde{X}_{1} \sim \tilde{\pi}$,

$$
\bar{Y}_{n}^{\mathrm{RB}}(f):=\frac{\sum_{i=1}^{n} f\left(\tilde{X}_{i}\right) / \varrho\left(\tilde{X}_{i}\right)}{\sum_{i=1}^{n} 1 / \varrho\left(\tilde{X}_{i}\right)}, \quad \bar{Y}_{n}^{\mathrm{Geo}}(f):=\frac{\sum_{i=1}^{n} \tau_{i} f\left(\tilde{X}_{i}\right)}{\sum_{i=1}^{n} \tau_{i}}, \quad n \geq 1,
$$

which are the two extremes considered by Douc and Robert (2011), whose Lemma 1 shows that the numerator of $\bar{Y}_{n}^{\mathrm{RB}}(f)$ is a Rao-Blackwellized version of the numerator of $\bar{Y}_{n}^{\mathrm{Geo}}(f)$ (see also Lemma 8 below): indeed a key observation is that $\tau_{i}$ is a Geometric $\left(\varrho\left(\tilde{X}_{i}\right)\right)$ random variable whose expectation is therefore $1 / \varrho\left(\tilde{X}_{i}\right)$. The following result summarizes the comparison between these two estimators, and is a special case of Douc and Robert [(2011), Theorem 1] but whose assumptions on $f$ are explicit.

Proposition 5. Let $f \in L^{1}(\pi), \bar{f}:=f-\pi(f), \bar{f} / \varrho \in L_{0}^{2}(\mathrm{E}, \tilde{\pi})$ and $\operatorname{var}(\bar{f} / \varrho, \tilde{P})<\infty$. Then:

1. $\bar{Y}_{n}^{\mathrm{RB}}(f) \stackrel{\text { a.s. }}{\rightarrow} \pi(f)$ and $\bar{Y}_{n}^{\mathrm{Geo}}(f) \stackrel{\text { a.s. }}{\rightarrow} \pi(f)$ as $n \rightarrow \infty$.

2. $\sqrt{n}\left[\bar{Y}_{n}^{\mathrm{RB}}(f)-\pi(f)\right] \stackrel{L}{\rightarrow} N\left(0, \sigma_{\mathrm{RB}}^{2}(f)\right)$, where

$$
\sigma_{\mathrm{RB}}^{2}(f)=\pi(\varrho)^{2} \operatorname{var}(\tilde{P}, \bar{f} / \varrho) .
$$

3. $\sqrt{n}\left[\bar{Y}_{n}^{\mathrm{Geo}}(f)-\pi(f)\right] \stackrel{L}{\rightarrow} N\left(0, \sigma_{\mathrm{Geo}}^{2}(f)\right)$, where

$$
\sigma_{\mathrm{Geo}}^{2}(f)=\pi(\varrho)\left\{\pi\left(\bar{f}^{2} / \varrho\right)-\pi\left(\bar{f}^{2}\right)+\pi(\varrho) \operatorname{var}(\tilde{P}, \bar{f} / \varrho)\right\} .
$$


It is clear that $\sigma_{\mathrm{RB}}^{2}(f) \leq \sigma_{\mathrm{Geo}}^{2}(f)$ for all appropriate $f$, and this motivated Douc and Robert (2011) to propose intermediate estimators with limiting variance in between $\sigma_{\mathrm{RB}}^{2}(f)$ and $\sigma_{\mathrm{Geo}}^{2}(f)$. Remark 7, however, shows that the computational benefits of such estimators are large only when the computational cost of obtaining the improved estimates is considerably less than that of simulating the chain itself.

REMARK 7. When

$$
\operatorname{var}(\tilde{P}, \bar{f} / \varrho) \geq \tilde{\pi}\left(\bar{f}^{2} / \varrho^{2}\right)=\pi\left(\bar{f}^{2} / \varrho\right) / \pi(\varrho),
$$

then $\pi(\varrho)^{2} \operatorname{var}(\tilde{P}, \bar{f} / \varrho) \geq \pi(\varrho) \pi\left(\bar{f}^{2} / \varrho\right)$ and so $\sigma_{\mathrm{Geo}}^{2}(f) \leq 2 \sigma_{\mathrm{RB}}^{2}(f)$. We note that (16) holds, for example, when $\tilde{P}$ is a positive operator on $L_{0}^{2}(\mathrm{E}, \tilde{\pi})$.

In order to prove Proposition 5, we first consider the sequences of unbiased estimators, with $\tilde{X}_{1} \sim \tilde{\pi}$, given by

$$
Y_{n}^{\mathrm{RB}}(f):=\frac{\pi(\varrho)}{n} \sum_{i=1}^{n} \frac{f\left(\tilde{X}_{i}\right)}{\varrho\left(\tilde{X}_{i}\right)}, \quad Y_{n}^{\mathrm{Geo}}(f):=\frac{\pi(\varrho)}{n} \sum_{i=1}^{n} \tau_{i} f\left(\tilde{X}_{i}\right), \quad n \geq 1 .
$$

Lemma 8. Let $f / \varrho \in L^{2}(\mathrm{E}, \tilde{\pi})$ and $\operatorname{var}(f / \varrho, \tilde{P})<\infty$. Then:

1. $Y_{n}^{\mathrm{RB}}(f) \stackrel{\text { a.s. }}{\rightarrow} \pi(f)$ as $n \rightarrow \infty$ and $Y_{n}^{\mathrm{Geo}}(f) \stackrel{\text { a.s. }}{\rightarrow} \pi(f)$ as $n \rightarrow \infty$.

2. Their asymptotic variances are

$$
\lim _{n \rightarrow \infty} n \operatorname{var}\left(Y_{n}^{\mathrm{RB}}(f)\right)=\pi(\varrho)^{2} \operatorname{var}(f / \varrho, \tilde{P}),
$$

and

(18) $\lim _{n \rightarrow \infty} n \operatorname{var}\left(Y_{n}^{\mathrm{Geo}}(f)\right)=\pi(\varrho) \pi\left(f^{2} / \varrho\right)-\pi(\varrho) \pi\left(f^{2}\right)+\pi(\varrho)^{2} \operatorname{var}(f / \varrho, \tilde{P})$.

PROOF. The first part follows from the Markov chain law of large numbers. For the second part, (17) follows from the definition of $\operatorname{var}(f / \varrho, \tilde{P})$. For (18), we apply the law of total variance

$$
n \operatorname{var}\left(Y_{n}^{\mathrm{Geo}}(f)\right)=n \mathbb{E}\left[\operatorname{var}\left(Y_{n}^{\mathrm{Geo}}(f) \mid \tilde{X}\right)\right]+n \operatorname{var}\left(\mathbb{E}\left[Y_{n}^{\mathrm{Geo}} \mid \tilde{X}\right]\right),
$$

and observe that for any $n \in \mathbb{N}$,

$$
\begin{aligned}
n \mathbb{E}\left[\operatorname{var}\left(Y_{n}^{\mathrm{Geo}}(f) \mid \tilde{X}\right)\right] & =n \pi(\varrho)^{2} \mathbb{E}\left[\operatorname{var}\left(\frac{1}{n} \sum_{i=1}^{n} \tau_{i} f\left(\tilde{X}_{i}\right) \mid \tilde{X}\right)\right] \\
& =n \pi(\varrho)^{2} \mathbb{E}\left[\frac{1}{n^{2}} \sum_{i=1}^{n} f\left(\tilde{X}_{i}\right)^{2} \operatorname{var}\left(\tau_{i} \mid \tilde{X}_{i}\right)\right]
\end{aligned}
$$




$$
\begin{aligned}
& =\pi(\varrho)^{2} \mathbb{E}\left[\frac{1}{n} \sum_{i=1}^{n} f\left(\tilde{X}_{i}\right)^{2} \frac{1-\varrho\left(\tilde{X}_{i}\right)}{\varrho\left(\tilde{X}_{i}\right)^{2}}\right] \\
& =\pi(\varrho)^{2} \tilde{\pi}\left(f^{2} \cdot(1-\varrho) / \varrho^{2}\right) \\
& =\pi(\varrho) \pi\left(f^{2} \cdot(1-\varrho) / \varrho\right),
\end{aligned}
$$

while

$$
\begin{aligned}
n \operatorname{var}\left(\mathbb{E}\left[Y_{n}^{\mathrm{Geo}}(f) \mid \tilde{X}\right]\right) & =n \operatorname{var}\left(\mathbb{E}\left[\frac{\pi(\varrho)}{n} \sum_{i=1}^{n} \tau_{i} f\left(\tilde{X}_{i}\right) \mid \tilde{X}\right]\right) \\
& =n \operatorname{var}\left(\frac{\pi(\varrho)}{n} \sum_{i=1}^{n} f\left(\tilde{X}_{i}\right) / \varrho\left(\tilde{X}_{i}\right)\right) \\
& =n \operatorname{var}\left(Y_{n}^{\mathrm{RB}}(f)\right),
\end{aligned}
$$

and the result follows from (17) by taking limits.

Proof OF Proposition 5. The first part follows from the Markov chain law of large numbers applied to $\frac{1}{n} \sum_{i=1}^{n} f\left(\tilde{X}_{i}\right) / \varrho\left(\tilde{X}_{i}\right), \frac{1}{n} \sum_{i=1}^{n} 1 / \varrho\left(\tilde{X}_{i}\right)$, $\frac{1}{n} \sum_{i=1}^{n} \tau_{i} f\left(\tilde{X}_{i}\right)$ and $\frac{1}{n} \sum_{i=1}^{n} \tau_{i}$. The second part follows from

$$
\begin{aligned}
\sqrt{n}\left[\bar{Y}_{n}^{\mathrm{RB}}(f)-\pi(f)\right] & =\sqrt{n}\left[\frac{\sum_{i=1}^{n} f\left(\tilde{X}_{i}\right) / \varrho\left(\tilde{X}_{i}\right)}{\sum_{i=1}^{n} 1 / \varrho\left(\tilde{X}_{i}\right)}-\pi(f)\right] \\
& =\sqrt{n}\left[\frac{\sum_{i=1}^{n} \bar{f}\left(\tilde{X}_{i}\right) / \varrho\left(\tilde{X}_{i}\right)}{\sum_{i=1}^{n} 1 / \varrho\left(\tilde{X}_{i}\right)}\right] \\
& =\frac{\frac{1}{\sqrt{n}} \pi(\varrho) \sum_{i=1}^{n} \bar{f}\left(\tilde{X}_{i}\right) / \varrho\left(\tilde{X}_{i}\right)}{\pi(\varrho) \frac{1}{n} \sum_{i=1}^{n} 1 / \varrho\left(\tilde{X}_{i}\right)}
\end{aligned}
$$

where the denominator converges almost surely to 1 by the Markov chain law of large numbers and the numerator converges weakly to a mean 0 normal random variable with variance $\sigma_{\mathrm{RB}}^{2}(f)$ by Lemma 8 and Häggström and Rosenthal [(2007), Corollary 6]; the result follows from Slutsky's lemma. For the third part, similar to the second part we obtain

$$
\sqrt{n}\left[\bar{Y}_{n}^{\mathrm{Geo}}(f)-\pi(f)\right]=\frac{\frac{1}{\sqrt{n}} \pi(\varrho) \sum_{i=1}^{n} \tau_{i} \bar{f}\left(\tilde{X}_{i}\right)}{\pi(\varrho) \frac{1}{n} \sum_{i=1}^{n} \tau_{i}},
$$

where the denominator converges almost surely to 1 by the Markov chain law of large numbers and the numerator converges weakly to a mean 0 normal random variable with variance $\sigma_{\text {Geo }}^{2}(f)$ by Lemma 8 and Häggström and Rosenthal [(2007), Corollary 6]; the result follows from Slutsky's lemma. 
6. Concluding remarks. We have exploited the relationship between a reversible Markov chain $X$ and its jump chain $\tilde{X}$ to characterize which ergodic averages of the two chains have finite asymptotic variance. This allows one to determine which ergodic averages of $X$ have finite asymptotic variance when $\tilde{X}$ is variance bounding. For independence samplers, this leads to a complete characterization of which ergodic averages have finite asymptotic variance. In the case of pseudo-marginal Markov chains, we provide a simple sufficient condition for all ergodic averages of $L^{2}$ functions of the primary variable to have finite asymptotic variance, even in situations where the pseudo-marginal chain is not variance bounding. For example, if the second moments of the noise variables are uniformly bounded but the noise variables themselves are not almost surely bounded then the pseudo-marginal Markov chain cannot be variance bounding [Andrieu and Roberts (2009), Andrieu and Vihola (2015)] and so at least some $L^{2}$ functions do not have a finite associated asymptotic variance [Roberts and Rosenthal (2008)]: it follows from Theorem 3 that these cannot be functions of the primary variable alone.

Our pseudo-marginal results rely on the marginal chain or its jump chain being variance bounding. In some cases, this may be verified using results in Roberts and Rosenthal (2008) and references therein. Verifying whether a reversible Markov chain is variance bounding in many practical situations will involve determining if it is geometrically ergodic, for which Foster-Lyapunov drift criteria [see, e.g., Roberts and Rosenthal (2004), Theorem 9] can be used: for Metropolis chains, the results of Jarner and Hansen (2000) are quite general. In practice, Theorem 3 allows one to determine that asymptotic variances of appropriate functions associated with the pseudo-marginal chain are finite by separately determining that the marginal chain is variance bounding, and that the uniformly bounded second moment condition on the noise variables is satisfied.

Acknowledgements. We are grateful to the referees for helpful comments that have improved the paper.

\section{REFERENCES}

Andrieu, C. and Roberts, G. O. (2009). The pseudo-marginal approach for efficient Monte Carlo computations. Ann. Statist. 37 697-725. MR2502648

ANDrieu, C. and Vihola, M. (2015). Convergence properties of pseudo-marginal Markov chain Monte Carlo algorithms. Ann. Appl. Probab. 25 1030-1077. MR3313762

ANDRIEU, C. and ViholA, M. (2016). Establishing some order amongst exact approximations of MCMCs. Ann. Appl. Probab. 26 2661-2696. MR3563190

Banterle, M., Grazian, C., Lee, A. and Robert, C. P. (2015). Accelerating MetropolisHastings algorithms by delayed acceptance. Available at arXiv:1503.00996.

Beaumont, M. A. (2003). Estimation of population growth or decline in genetically monitored populations. Genetics $1641139-1160$.

BEDNORZ, W., ŁATUSZYŃSKI, K. and LATAŁA, R. (2008). A regeneration proof of the central limit theorem for uniformly ergodic Markov chains. Electron. Commun. Probab. 13 85-98.

Bornn, L., Pillai, N., Smith, A. and Woodard, D. (2017). The use of a single pseudo-sample in approximate Bayesian computation. Stat. Comput. 27 583-590. 
Caracciolo, S., Pelissetto, A. and Sokal, A. D. (1990). Nonlocal Monte Carlo algorithm for self-avoiding walks with fixed endpoints. J. Stat. Phys. 60 1-53.

CostA, O. L. V. (1990). Stationary distributions for piecewise-deterministic Markov processes. J. Appl. Probab. 27 60-73.

Costa, O. L. V. and Dufour, F. (2008). Stability and ergodicity of piecewise deterministic Markov processes. SIAM J. Control Optim. 47 1053-1077.

Douc, R. and RoBert, C. P. (2011). A vanilla Rao-Blackwellization of Metropolis-Hastings algorithms. Ann. Statist. 39 261-277. MR2797846

Doucet, A., Pitt, M. K., Deligiannidis, G. and Kohn, R. (2015). Efficient implementation of Markov chain Monte Carlo when using an unbiased likelihood estimator. Biometrika 102 295313.

GeYer, C. J. (1992). Practical Markov chain Monte Carlo. Statist. Sci. 7 473-483.

HÄGGSTRÖM, O. and Rosenthal, J. S. (2007). On variance conditions for Markov chain CLTs. Electron. Commun. Probab. 12 454-464.

Hastings, W. K. (1970). Monte Carlo sampling methods using Markov chains and their applications. Biometrika 57 97-109.

JARNER, S. F. and HANSEN, E. (2000). Geometric ergodicity of Metropolis algorithms. Stochastic Process. Appl. 85 341-361. MR1731030

JARner, S. F. and Roberts, G. O. (2002). Polynomial convergence rates of Markov chains. Ann. Appl. Probab. 12 224-247. MR1890063

JARneR, S. F. and RoberTs, G. O. (2007). Convergence of heavy-tailed Monte Carlo Markov chain algorithms. Scand. J. Stat. 34 781-815. MR2396939

Kipnis, C. and Varadhan, S. R. S. (1986). Central limit theorem for additive functionals of reversible Markov processes and applications to simple exclusions. Comm. Math. Phys. $1041-$ 19.

LEE, A. and ŁATUSZYŃSKI, K. (2014). Variance bounding and geometric ergodicity of Markov chain Monte Carlo kernels for approximate Bayesian computation. Biometrika 101 655-671.

Lin, L., LiU, K. F. and SlOAN, J. (2000). A noisy Monte Carlo algorithm. Phys. Rev. D 61074505.

Mengersen, K. L. and Tweedie, R. L. (1996). Rates of convergence of the Hastings and Metropolis algorithms. Ann. Statist. 24 101-121. MR1389882

Metropolis, N., Rosenbluth, A. W., Rosenbluth, M. N., Teller, A. H. and Teller, E. (1953). Equation of state calculations by fast computing machines. J. Chem. Phys. 21 1087-1092.

Meyn, S. and Tweedie, R. L. (2009). Markov Chains and Stochastic Stability, 2nd ed. Cambridge Univ. Press, Cambridge. MR2509253

PARdouX, E. (2008). Markov Processes and Applications. Wiley, New York.

PESKUn, P. H. (1973). Optimum Monte-Carlo sampling using Markov chains. Biometrika $60607-$ 612.

Roberts, G. O. and Rosenthal, J. S. (2004). General state space Markov chains and MCMC algorithms. Probab. Surv. $120-71$.

Roberts, G. O. and Rosenthal, J. S. (2008). Variance bounding Markov chains. Ann. Appl. Probab. 18 1201-1214.

Sherlock, C., Thiery, A. H. and Lee, A. (2017). Pseudo-marginal Metropolis-Hastings sampling using averages of unbiased estimators. Biometrika 104 727-734.

Sherlock, C., Thiery, A. H., Roberts, G. O. and Rosenthal, J. S. (2015). On the efficiency of pseudo-marginal random walk Metropolis algorithms. Ann. Statist. 43 238-275. MR3285606

TAVARÉ, S., BALDing, D. J., GRIFFiths, R. C. and DonNelly, P. (1997). Inferring coalescence times from DNA sequence data. Genetics 145 505-518.

TIERNEY, L. (1994). Markov chains for exploring posterior distributions. Ann. Statist. 22 17011762. MR1329166

Tierney, L. (1998). A note on Metropolis-Hastings kernels for general state spaces. Ann. Appl. Probab. 8 1-9. MR1620401 
YosidA, K. (1980). Functional Analysis, 6th ed. Springer, Berlin.

DEPARTMENT OF STATISTICS

UNIVERSITY OF OXFORD

24-29 ST. GILES

OXFORD OX1 3LB

UNITED KINGDOM

E-MAIL: deligian@stats.ox.ac.uk
DEPARTMENT OF STATISTICS

UNIVERSITY OF WARWICK

COVENTRY CV4 7AL

UNITED KINGDOM

AND

SCHOOL OF MATHEMATICS

UNIVERSITY OF BRISTOL

UNIVERSITY WALK

BRISTOL BS8 1TW

UNITED KINGDOM

AND

The Alan Turing Institute

BRITISH LIBRARY

96 EUSTON ROAD

LONDON NW1 2DB

UNITED KINGDOM

E-MAIL: anthony.lee@bristol.ac.uk 